\title{
Vortex dynamics and Hall conductivity of hard-core bosons
}

\author{
Netanel Lindner, ${ }^{1,2}$ Assa Auerbach, ${ }^{1}$ and Daniel P. Arovas ${ }^{3}$ \\ ${ }^{1}$ Physics Department, Technion, 32000 Haifa, Israel \\ ${ }^{2}$ Institute of Quantum Information, California Institute of Technology, Pasadena, California 91125, USA \\ ${ }^{3}$ Department of Physics, University of California at San Diego, La Jolla, California 92093, USA \\ (Received 9 June 2010; revised manuscript received 22 July 2010; published 7 October 2010)
}

\begin{abstract}
Magnetotransport of hard-core bosons is studied using an $X X Z$ quantum spin model representation, appropriately gauged on the torus to allow for an external magnetic field. We find strong lattice effects near half filling. An effective quantum mechanical description of the vortex degrees of freedom is derived. Using semiclassical and numerical analysis we compute the vortex-hopping energy $t_{\mathrm{V}}$, which at half filling is close to magnitude of the boson hopping energy. The critical quantum melting density of the vortex lattice is estimated at $6.5 \times 10^{-3}$ vortices per unit cell. The Hall conductance is computed from the Chern numbers of the lowenergy eigenstates. At zero temperature, it reverses sign abruptly at half filling. At precisely half filling, all eigenstates are doubly degenerate for any odd number of flux quanta. We prove the exact degeneracies on the torus by constructing an $\mathrm{SU}(2)$ algebra of point-group symmetries, associated with the center of vorticity. This result is interpreted as if each vortex carries an internal spin-half degree of freedom, which can manifest itself as a charge density modulation in its core. Our findings suggest interesting experimental implications for vortex motion of cold atoms in optical lattices and magnet transport of short coherence length superconductors.
\end{abstract}

DOI: $10.1103 /$ PhysRevB.82.134510

PACS number(s): 05.30.Jp, 03.75.Lm, 66.35.+a

\section{INTRODUCTION}

Hard-core bosons (HCB) are often used to describe superfluids and superconductors which are characterized by low superfluid stiffness and short coherence lengths. As such, HCB are relevant to cold atomic gases in optical lattices, ${ }^{1,2}$ low capacitance Josephson junction arrays, ${ }^{3-5}$ disordered superconducting films, ${ }^{6}$ and cuprate superconductors. ${ }^{7-13}$

At low densities, HCB can be treated by weak-coupling (Bogoliubov) perturbation theory. ${ }^{14}$ Closer to half filling, lattice umklapp scattering and the hard-core constraints become important. Recent calculations of the dynamical conductivity of HCB near half filling ${ }^{15}$ demonstrate the breakdown of weak-scattering Drude-Boltzmann transport theory in this regime. HCB exhibit so-called "bad metal" phenomenology, (i.e., large resistivity, linearly increasing in temperature). Such behavior has been often observed in unconventional (short coherence length) superconductors. ${ }^{16}$

This paper concerns dynamical correlations of HCB and their vortices near half filling. These will be exposed by including a weak orbital magnetic field in the Hamiltonian and studying the Hall effect.

Our primary results are as follows. First, we apply a combination of semiclassical analysis and exact diagonalization to the gauged XXZ Hamiltonian on a finite lattice on the torus. We highlight the (sometimes overlooked) fact that a uniform magnetic field of one flux quantum penetrating the surface of the torus beaks translational symmetry. As a consequence, the semiclassical vortex center is subjected to a confining potential minimized at a well-defined position. Fitting the low many-body spectrum to an effective singlevortex Hamiltonian, we determine the vortex hopping rate (effective mass).

Near half filling, the vortex mass is found to be similar in magnitude to the HCB mass. This allows us to estimate the critical field for quantum melting of the vortex solid (super- fluid) phase at $6.5 \times 10^{-4}$ flux quanta per unit cell.

Second, at half filling we find doublet degeneracies associated with an odd number of magnetic flux quanta penetrating the torus. We associate them with symmetries about the vortex position and label the emergent degrees of freedom as "vortex spin" (v-spin). Physically, these degrees of freedom correspond to the orientation of the charge density wave in the vortex cores.

Finally, we compute the Hall conductivity using thermally averaged Chern numbers. In stark contrast to continuum bosons, and to electrons in metallic bands, we find that the Hall conductivity of HCB reverses sign abruptly at half filling. The associated Hall temperature scale vanishes at half filling, signaling a possible quantum phase transition for the thermodynamic system in a magnetic field. Some of these results were briefly reported in a recent letter. ${ }^{17}$

This paper is organized as follows. In Sec. II the HCB Hamiltonian is introduced, with a discussion of its charge conjugation symmetry about half filling. Semiclassical approximations are derived in Sec. III, for the various regimes of filling. At low density, we recover the Gross-Pitaevskii (GP) theory with its Galilean invariant vortex dynamics and classical Hall effect. At half filling, the continuum limit corresponds to the anisotropic gauged nonlinear sigma model. Its vortices possess localized charge density waves in their cores. Section IV describes the mathematical peculiarities of the gauged torus, including translational symmetry breaking (TSB) (elaborated in Appendix). Definitions of null lines, null points, and vorticity centers are provided. The point group symmetry generators $\Pi_{V}^{x}$ and $\Pi_{V}^{x}$ are constructed and their commutator is calculated. The proof of $\mathrm{v}$-spin degeneracies at half filling is provided. Section V computes the vortex effective hamiltonian by combining semiclassical and exact diagonalization calculations. The critical field for quantum melting of the vortex lattice is deduced from our value of vortex hopping rate. Section VII computes the Hall con- 
ductance on the torus as a function of density and temperature. We conclude in Sec. VIII and discuss experimental implications of our results in cold atoms and cuprate superconductors.

\section{HARD-CORE BOSONS}

The conventional Bose Hubbard model for interacting lattice bosons is

$$
\begin{aligned}
\mathcal{H}_{U}= & -2 J \sum_{\langle i j\rangle}\left(e^{i q A_{i j}} a_{i}^{\dagger} a_{j}+a^{-i q A_{i j}} a_{j}^{\dagger} a_{i}\right) \\
& +4 V \sum_{\langle i j\rangle}\left(n_{i}-\frac{1}{2}\right)\left(n_{j}-\frac{1}{2}\right)-\mu \sum_{i} n_{i}+\frac{1}{2} U \sum_{i} n_{i}\left(n_{i}-1\right) .
\end{aligned}
$$

In the hard-core limit $U \rightarrow \infty$, Eq. (1) reduces to the HCB Hamiltonian as $\mathcal{H}=\mathcal{P H}_{U=0} \mathcal{P}$, where $\mathcal{P}$ is the projector onto the subspace where $n_{i}=0$ or 1 for each site.

We use units where $\hbar=c=1$. $\langle i j\rangle$ denotes a nearest neighbor link on the square lattice; the lattice constant is $a=1 . J$ is the Josephson coupling, $q$ is the boson charge and $A_{i j}$ the electromagnetic gauge field on a bond. $V$ is a nearest neighbor repulsive interaction. In the $\mathrm{HCB}$ limit, The chemical potential $\mu=0$ corresponds to a density of half filling $\langle n\rangle$ $=\frac{1}{2}$ operators, with half a boson per site on average.

As is well known, HCB operators obey an algebra corresponding to spin $\frac{1}{2}$ operators

$$
\begin{gathered}
\tilde{a}_{i}^{\dagger}=\mathcal{P} a_{i}^{\dagger} \mathcal{P}=S_{i}^{+}, \\
\tilde{a}_{i}=\mathcal{P} a_{i} \mathcal{P}=S_{i}^{-}, \\
n_{i}=\widetilde{a}_{i}^{\dagger} \widetilde{a}_{i}=S_{i}^{z}+\frac{1}{2} .
\end{gathered}
$$

By $\left[S_{i}^{+}, S_{j}^{-}\right]=2 S_{i}^{z} \delta_{i j}$, HCB operators obey constrained commutation relations,

$$
\left[\tilde{a}_{i}, \tilde{a}_{j}^{\dagger}\right]=\left(1-2 n_{i}\right) \delta_{i j}
$$

The constraint corrections $-2 n_{i} \delta_{i j}$ become important near half filling. $\lim _{U \rightarrow \infty} \mathcal{H}_{U}$ is thus represented by the gauged spin-half quantum $X X Z$ model,

$$
\begin{aligned}
\mathcal{H}= & -2 J \sum_{\langle i j\rangle}\left(e^{i q A_{i j}} S_{i}^{+} S_{j}^{-}+e^{-i q A_{i j}} S_{i}^{-} S_{j}^{+}\right)+4 V \sum_{\langle i, j\rangle} S_{i}^{z} S_{j}^{z} \\
& -\mu \sum_{i}\left(S_{i}^{z}+\frac{1}{2}\right) .
\end{aligned}
$$

It is widely believed that the two-dimensional ground state of Eq. (4), in the thermodynamic limit, exhibits magnetic order. In the regime of $V \ll J$, which is relevant to this paper, the ordered moment lies in the $X Y$ plane, $\left\langle S_{i}^{+}\right\rangle \neq 0$. That is to say, except for the limits $n=0,1$, the ground state of $\mathrm{HCB}$ is expected to exhibit long-range superfluid order.

\section{HCB charge conjugation symmetry}

An important distinction between the HCB Hamiltonian (4) and the finite $U$ Bose-Hubbard model of Eq. (1), is the emergence of charge conjugation symmetry in the infinite $U$ limit. One defines the unitary charge conjugation operator,

$$
C \equiv \exp \left(i \pi \sum_{i} S_{i}^{x}\right)
$$

$C$ transforms "particles" into "holes," i.e., $C^{\dagger} \tilde{n}_{i} C=1-\tilde{n}_{i}$, and

$$
C^{\dagger} \mathcal{H}(q A, \mu) C=\mathcal{H}(-q A,-\mu) .
$$

At half filling $(\mu=0)$, and $\boldsymbol{A}=0$, the Hamiltonian is invariant under charge conjugation on any lattice structure. ${ }^{18}$

A consequence of Eq. (6) is that the Hall conductivity (which is linear in $q$ ) is antisymmetric in the deviation from half filling, i.e.,

$$
\sigma_{x y}(n, T)=-\sigma_{x y}(1-n, T) .
$$

In contrast, the superfluid stiffness $\rho_{s}(n)$ and longitudinal conductivity $\sigma_{x x}(n)$ are symmetric under $n \rightarrow(1-n)$.

In terms of vortex motion, Eq. (7) implies that below and above half-filling vortices drift in opposite directions relative to the particle current.

\section{SEMICLASSICAL THEORY}

The partition function of HCB can be represented by the spin half coherent state path integral, ${ }^{19,20}$

$$
Z=\int \mathcal{D} \hat{\mathbf{\Omega}}(\tau) \exp \left[\int_{0}^{\beta} d \tau\left(i K-H^{c l}\right)\right],
$$

where

$$
\begin{gathered}
K[\hat{\mathbf{\Omega}}, \dot{\hat{\mathbf{\Omega}}}] \equiv \frac{1}{2} \sum_{i}\left(1-\cos \theta_{i}\right) \dot{\boldsymbol{\phi}}_{i} \\
H^{\mathrm{cl}}[\hat{\mathbf{\Omega}}, \boldsymbol{A}]=-J \sum_{\langle i, j\rangle} \sin \theta_{i} \sin \theta_{j} \cos \left(\phi_{i}-\phi_{j}+q A_{i j}\right) \\
+V \sum_{\langle i, j\rangle} \cos \theta_{i} \cos \theta_{j}-\frac{\mu}{2} \sum_{i} \cos \theta_{i} .
\end{gathered}
$$

$\hat{\mathbf{\Omega}}_{i}=\left(\theta_{i}, \phi_{i}\right)$ are the polar angles on a sphere. The spin size $S=\frac{1}{2}$ plays the role of the large parameter which controls the semiclassical expansion.

In the classical (saddle point) approximation, for $\boldsymbol{A}=0$, the ground state superfluid stiffness is

$$
\begin{aligned}
\rho_{\mathrm{s}}^{\mathrm{cl}} & =\left.q^{-2} \frac{\partial^{2} H^{\mathrm{cl}}}{\partial A_{\boldsymbol{r}, \boldsymbol{r}+\hat{x}}^{2}}\right|_{A=0} \\
& =J\left\langle\sin ^{2} \theta_{\boldsymbol{r}}\right\rangle=4 J n(1-n)
\end{aligned}
$$

which (in contrast to continuum bosons) exhibits a nonmonotonic dependence on $n$. At half filling (optimal density), $\rho_{\mathrm{s}}$ is maximized. Quantum corrections enhance $\rho_{\mathrm{s}}^{\mathrm{cl}}\left(n=\frac{1}{2}\right)$ further by about $7 \% .^{21,22}$ The superfluid stiffness vanishes at the Berezinskii-Kosterlitz-Thouless (BKT) (Ref. 23) transition temperature, computed to be $T_{\mathrm{BKT}} \simeq 1.41 J$.

The kinetic term $K$ of Eq. (8), determines the quantum dynamics. The harmonic spin-wave expansion of Eq. (10) 
yields a linearly dispersing phase fluctuations mode. The order parameter is suppressed to zero at all finite temperatures, in accordance with the Mermin-Wagner theorem.

\section{A. Low-density, Gross-Pitaevskii limit}

For large negative values of the chemical potential $\mu$, the action in Eq. (10) can be expanded around the ferromagnetic (low-density) state of $\theta_{i} \approx \pi$,

$$
\cos \theta_{i} \rightarrow 2 n_{i}-1, \quad \sin \theta_{i} \approx 2 \sqrt{n_{i}\left(1-n_{i}\right)} .
$$

We define the continuous field

$$
\psi\left(\boldsymbol{x}_{i}\right)=\sqrt{n_{i}} e^{i \phi_{i}},
$$

and replace the measure by

$$
\prod_{i} \mathcal{D} \cos \theta_{i} \mathcal{D} \phi_{i} \rightarrow \mathcal{D} \psi^{*} \mathcal{D} \psi \prod_{i, t} \Theta\left(1-\int_{V_{i}} d^{2} x \psi^{*} \psi\right),
$$

up to an unnecessary normalization constant. The Heaviside functions enforce the hard-core constraint $n_{i} \leq 1$ in the $i$ th unit cell at each time slice. In the low-density limit, these constraints are ignored, and the action Eq. (10) is expanded to leading order in $n_{i} \ll \frac{1}{2}$, and gradients $\nabla \psi$. This yields an effective GP theory, ${ }^{1}$

$$
\begin{gathered}
Z_{\mathrm{GP}}=\int \mathcal{D} \psi^{*} \mathcal{D} \psi \exp \left(-S_{\mathrm{GP}}\left[\psi^{*}, \psi, \boldsymbol{A}\right]+\cdots\right) \\
S_{\mathrm{GP}}=\int d^{2} x \int d t\left[\psi^{*}\left(\partial_{t}-\mu\right) \psi+\frac{1}{2 m^{*}}|(-i \nabla-q \boldsymbol{A}) \psi|^{2}\right. \\
\left.+\frac{1}{2} g|\psi|^{4}\right],
\end{gathered}
$$

where the effective mass and interaction parameters are given by

$$
\begin{gathered}
m^{*}=\frac{1}{2 J} \\
g=16(J+V) .
\end{gathered}
$$

In the presence of a magnetic field $B \hat{z}$, a density of $n_{\mathrm{v}}$ $=B / \phi_{0}$ vortices is produced, where $\phi_{0}=2 \pi / q$ is the flux quantum. The core profile function $|\psi(\boldsymbol{r})|=f\left(\boldsymbol{r}-\boldsymbol{R}_{j}\right)$ near vortex $j$ is well approximated by minimizing the GP energy, which yields ${ }^{1}$

$$
f_{\mathrm{GP}}(\boldsymbol{r}) \simeq \frac{\sqrt{n} r}{\sqrt{\xi^{2}+r^{2}}},
$$

where $\xi=1 / \sqrt{g m^{*} n}$ is the coherence length. For $n \ll \frac{1}{2}$, one has $\xi \gg a$. The core density depletion is proportional to $n$ $-\left|f_{\mathrm{GP}}\right|^{2}$. Hence it decays as $1 / r^{2}$ away from the vortex center.

In the high-density limit, $n \rightarrow 1$, the partition function can also be approximated by the same GP action [Eq. (15)] following a particle hole transformation [Eq. (6)]. In this case, $|\psi|^{2}$ represents the density of holes.
By neglecting the higher order gradients and the hard-core constraints, the GP theory does not include lattice scattering effects as it is completely Galilean invariant. Consider an externally induced uniform current density

$$
\boldsymbol{j}=q n \boldsymbol{v}_{s} \text {. }
$$

In the moving frame of velocity $\boldsymbol{v}_{s}$ the vortices are stationary. Therefore, back in the laboratory frame, a purely transverse electromotive field is produced by the moving vortices,

$$
\begin{gathered}
\boldsymbol{E}=\frac{h}{q} \hat{z} \times \boldsymbol{j}_{\mathrm{v}} \\
=\frac{h}{q} n_{v} \hat{z} \times \boldsymbol{V}_{\mathrm{v}} \\
=\frac{h}{q^{2}} \frac{n_{v}}{n} \hat{z} \times \boldsymbol{j} .
\end{gathered}
$$

That is to say, in the pure GP theory, the longitudinal (dissipative) conductivity vanishes and the Hall conductivity equals to the classical value,

$$
\begin{gathered}
\sigma_{x x}=0, \\
\sigma_{x y}=\left(\frac{q^{2}}{h}\right)\left(\frac{n}{n_{v}}\right)=\frac{n q}{B} .
\end{gathered}
$$

Spoiling Galilean invariance by the presence of nonuniform potentials, boundary conditions, or by an underlying lattice can allow vortices to tunnel between different real space positions, resulting in a longitudinal conductivity. ${ }^{24,25}$

\section{B. Half-filling, anisotropic $\sigma$ model}

Toward half filling, lattice scattering modifies the vortex structure and dynamics. At half filling $\mu=0$, the semiclassical theory of Eq. (10) is described by the anisotropic nonlinear $\sigma$ model (NLSM). ${ }^{26}$ After a sublattice rotation $\Pi_{i \in B} e^{i \pi S_{i}^{z}}$ all the pseudospin interactions are antiferromagnetic. The spins $\hat{\mathbf{\Omega}}_{i}$ are represented by

$$
\hat{\boldsymbol{\Omega}}_{i}=\eta_{i} \hat{\boldsymbol{n}}\left(\boldsymbol{x}_{i}\right) \sqrt{1-\left[\boldsymbol{L}\left(\boldsymbol{x}_{i}\right) / \bar{S}\right]^{2}}+\boldsymbol{L}\left(\boldsymbol{x}_{i}\right) / \bar{S},
$$

where $\eta_{i}= \pm 1$ on the $A(+)$ and $B(-)$ sublattices, respectively. The Néel vector $\hat{\boldsymbol{n}}$ satisfies $\hat{\boldsymbol{n}}^{2}=1$ and is orthogonal to the local magnetization $\boldsymbol{L}$, i.e., $\hat{\boldsymbol{n}} \cdot \boldsymbol{L}=0$.

The complex combination

$$
n_{\perp}=n_{x}+i n_{y}=\left|n_{\perp}\right| e^{i \phi},
$$

defines the local superfluid order parameter, and $n_{z}$ corresponds to a bipartite charge density wave (CDW) with two possible signs. Following Refs. 19, 20, and 26, we substitute Eq. (21) in the measure and action of Eq. (10), and expand them to quadratic order in $\boldsymbol{L}$ and $\nabla \hat{\boldsymbol{n}}$. Integrating out $\boldsymbol{L}$ arrives at the anisotropic NLSM path integral,

$$
Z_{\mathrm{NLSM}}=\int \mathcal{D} \hat{\boldsymbol{n}} e^{i \mathrm{Y}[\hat{\boldsymbol{n}}]} e^{-S_{\mathrm{E}}[\hat{\boldsymbol{n}}, \boldsymbol{A}]},
$$

where $S_{\mathrm{E}}=\int_{0}^{\beta} d \tau \int d^{2} x \mathcal{L}_{\mathrm{E}}$ is the Euclidean action, with 


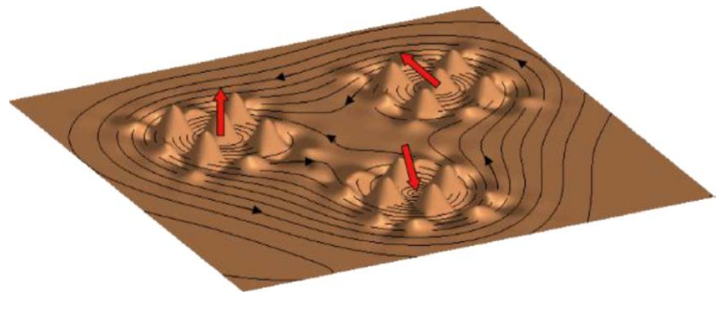

FIG. 1. (Color online) Illustration of three static vortices with their v-spins. Arrows describe the v-spin directions, their $z$ component is the local charge density wave near the vortex core, illustrated as ripples in the surface. Current density is depicted by black field lines.

$$
\begin{aligned}
\mathcal{L}_{\mathrm{E}}= & \frac{1}{2} \chi_{\perp}\left|\dot{n}_{\perp}\right|^{2}+\frac{1}{2} \chi_{z} \dot{n}_{z}^{2}+\frac{1}{2} \rho_{\mathrm{s}}\left|(\nabla-i q A) n_{\perp}\right|^{2}+\frac{1}{2} \rho_{\mathrm{s}}^{z}\left(\nabla n_{z}\right)^{2} \\
& +m_{z}^{2} n_{z}^{2}
\end{aligned}
$$

and

$$
\Upsilon=S \sum_{i} \eta_{i} \int_{0}^{\beta} d \tau\left(1-n_{z}\right) \dot{\phi}_{i}
$$

is the geometric Berry phase for spin $S$. The bare coupling constants are obtained directly from $H^{\mathrm{cl}}$ :

$$
\chi_{\perp}=\frac{S^{2}}{8 J}, \quad \chi_{z}=\frac{S^{2}}{4(J+V)},
$$

and

$$
\rho_{s}=J, \quad \rho_{s}^{z}=V, \quad m_{z}^{2}=2(J-V) .
$$

For $\boldsymbol{A}=0$, the isotropic (Heisenberg) limit is at $J=V, m_{z}$ $=0$. The Néel ground state implies degeneracy between superfluid and CDW order, and the existence of two massless Goldstone modes. At finite $X Y$ anisotropy, $m_{z}>0$, and there is one massless (phase) mode, and a gapped CDW (roton) mode at the CDW ordering wavevector $(\pi, \pi)$.

Vortex configurations at half filling can be viewed as a localized meron (half skyrmion) of the Néel field. Since $\left|\tilde{n}_{x y}\right|=0$ at the vortex center, and $n_{z}^{2}=1-\left|\tilde{n}_{x y}\right|^{2}$, the semiclassical vortex has a CDW in its core, as illustrated in Fig. 1.

Due to the finite anisotropy "mass" $m_{z}>0, n_{z}(\boldsymbol{r})$ decays exponentially away from the center

$$
\begin{gathered}
n_{\perp}(\boldsymbol{r})=\sqrt{1-n_{z}^{2}(\boldsymbol{r})} e^{i \phi(\boldsymbol{r})}, \\
n_{z}(\boldsymbol{r}) \sim e^{-r / \xi_{z}}, \\
\xi_{z}=\sqrt{\rho_{\mathrm{s}}^{z}} / m_{z} .
\end{gathered}
$$

Indeed variational calculations have previously shown that at half filling CDW ordering is found in the localized vortex core. ${ }^{27}$ In Sec. IV we shall show that the "orientation" of the charge density wave is actually a continuous SU(2) symmetry of the quantum Hamiltonian at half filling, which we name $v$-spin.

Since the system is charge conjugation symmetric at halffilling, there is no net charge depletion associated with the vortex core, and thus the statistical Berry phase for exchang- ing two vortices is zero. In other words, the vortices exhibit mutual Bose statistics. This is to be contrasted with GP vortices at low filling. As shown in Eq. (17), GP vortices involve a large density depletion (or accumulation, above half filling), which decays slowly away from their core. ${ }^{28}$

\section{Vortex hopping Hamiltonian}

In the limit where the number of lattice sites $N$ tends to infinity, the confining potential on the vortex (to be discussed shortly) vanishes, and the vortex energy is periodic on the lattice. Its minima lie in plaquette centers (i.e., at dual lattice sites). While the effects of the Berry phase $e^{i \Upsilon}$ in Eq. (27) are negligible for the static correlations in the superfluid phase, it is important for the dynamics of $\mathrm{HCB}$ and their vortices. The Berry phase determines the effective quantum Hamiltonian of vortices as follows.

When a vortex moves between dual lattice sites, the pathdependent geometric phase $Y$ yields $2 \pi$ times the number of bosons enclosed by the path. At half filling, this amounts to an effective $\pi$ flux per dual plaquette. These phases can be incorporated in an effective hopping model by the dual lattice gauge field $\mathcal{A}_{\boldsymbol{R}, \boldsymbol{R}+\boldsymbol{\eta}}$ along the link from site $\boldsymbol{R}$ to $\boldsymbol{R}+\boldsymbol{\eta}$. Thus for a single vortex on the infinite lattice, one can write an effective Harper Hamiltonian,

$$
\begin{aligned}
H_{\mathrm{V}}^{\infty}= & -\frac{1}{2} t_{\mathrm{V}} \sum_{\boldsymbol{R}, \boldsymbol{\eta}}\left(e^{\left.i \mathcal{A}_{\boldsymbol{R}, \boldsymbol{R}+\boldsymbol{\eta}} b_{\boldsymbol{R}}^{\dagger} b_{\boldsymbol{R}+\boldsymbol{\eta}}+\text { H.c. }\right)}\right. \\
& \sum_{\mathcal{C}} \mathcal{A}_{\boldsymbol{R}, \boldsymbol{R}+\boldsymbol{\eta}}=2 \pi \sum_{i \in \operatorname{int}(\mathcal{C})} n_{i},
\end{aligned}
$$

where the sum on the second line is a over a set of links comprising a closed path $\mathcal{C}$ on the dual lattice, and $\operatorname{int}(\mathcal{C})$ is the interior of this path, which consists of a set of sites on the original lattice bounded by $\mathcal{C}$.

\section{GAUGED TORUS}

We now return to the original HCB Hamiltonian, Eq. (4). We consider a finite square lattice, of dimensions $L_{x} \times L_{y}$, with $N=L_{x} L_{y}$ sites and periodic boundary conditions in both the $x$ and $y$ directions. This toroidal geometry is convenient for the study of finite lattices as it minimizes the effects of boundaries. It also provides external control over the positions of vortices via the two Aharonov-Bohm (AB) fluxes which run along the two cycles of the torus. The lattice site positions are labeled as

$$
\begin{aligned}
& x_{i}=0,1,2, \ldots, L_{x}-1 \\
& y_{i}=0,1,2, \ldots, L_{y}-1 .
\end{aligned}
$$

A uniform magnetic field $B$ is everywhere perpendicular to the surface, such that the total number of flux quanta penetrating the surface is $N_{\phi}=N B / \phi_{0}$, where $\phi_{0}=2 \pi / q$ is the flux quantum.

We construct a (piecewise differentiable) gauge field $\boldsymbol{A}(\boldsymbol{x})$ which interpolates the lattice gauge field on the surface of the torus, and obeys 


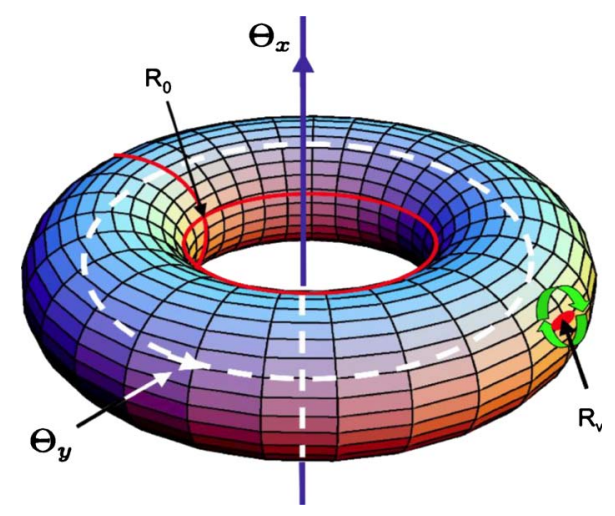

FIG. 2. (Color online) The gauged torus, which defines the finite size geometry of the HCB model. The torus surface is penetrated by a uniform magnetic field of one total flux quantum, and threaded by two Aharonov Bohm fluxes $\Theta=\left(\Theta^{x}, \Theta^{y}\right)$. Thick (red online) circles denote the null lines which enclose zero flux, and intersect at the null point $\boldsymbol{R}_{0}(\boldsymbol{\Theta})$. The vorticity center $\boldsymbol{R}_{\mathrm{V}}$ is located on the antipodal point to $\boldsymbol{R}_{0}$. The circulating currents of the ground state are illustrated by thick green arrows. This geometry is used to compute the vortex mass and Hall conductivity of $\mathrm{HCB}$, and to prove the v-spin degeneracies at half filling.

$$
\begin{gathered}
\hat{z} \cdot \nabla \times \boldsymbol{A}=B \\
\boldsymbol{\eta} \cdot \boldsymbol{A}\left(\boldsymbol{r}+\frac{1}{2} \boldsymbol{\eta}\right)=A_{\boldsymbol{r}, \boldsymbol{r}+\boldsymbol{\eta}},
\end{gathered}
$$

where $\boldsymbol{\eta}= \pm \hat{\boldsymbol{x}}, \pm \hat{\boldsymbol{y}} . \boldsymbol{A}$ determines the magnetic fluxes which flow through vertical and horizontal circumferences of the torus. These are given by the gauge invariant Wilson loop functions,

$$
\begin{aligned}
& W_{y}(x)=q \oint d y A_{y}(x, y) \bmod 2 \pi, \\
& W_{x}(y)=q \oint d x A_{x}(x, y) \bmod 2 \pi .
\end{aligned}
$$

The dimensionless AB parameters $\Theta=\left(\Theta_{x}, \Theta_{y}\right)$ are defined by the Wilson loops at $x=0$ and $y=0$,

$$
\begin{aligned}
& \Theta_{y}=W_{y}(x=0), \\
& \Theta_{x}=W_{x}(y=0) .
\end{aligned}
$$

$\boldsymbol{\Theta}$ lives on the reciprocal torus $[0,2 \pi) \times[0,2 \pi)$.

$W_{\alpha}=0, \alpha=x, y$ define the null lines on the torus. For $N_{\phi}=1$, there is one null line in each direction $x=X_{0}$, and $y$ $=Y_{0}$, as depicted in Fig. 2. Their intersection is the null point $\boldsymbol{R}_{0}=\left(X_{0}, Y_{0}\right)$, which constitutes a gauge invariant symmetry point on the torus.

$$
X_{0}(\Theta)=-\frac{L_{x} \Theta_{y}}{2 \pi},
$$

$$
Y_{0}(\Theta)=+\frac{L_{y} \Theta_{x}}{2 \pi} .
$$

The existence of a special point $\boldsymbol{R}_{0}$ on the torus, demonstrates the unintuitive fact that a uniform magnetic field necessarily destroys lattice translational symmetry. This fact is closely related to the quantization of Dirac monopoles in three dimensions. We elaborate further on this fact in Appendix. Equation (34) shows that $\boldsymbol{R}_{0}(\boldsymbol{\Theta})$ can be moved continuously on the torus by changing the AB parameters $\boldsymbol{\Theta}$. $^{29,30}$

As we shall see in Sec. V, semiclassical analysis and exact diagonalizations find that the center of vorticity $\boldsymbol{R}_{\mathrm{V}}$ is located at the antipodal position of the null point on the torus,

$$
\boldsymbol{R}_{\mathrm{V}}(\boldsymbol{\Theta})=\left[\frac{1}{2} L_{x}+X_{0}(\boldsymbol{\Theta}), \frac{1}{2} L_{y}+Y_{0}(\boldsymbol{\Theta})\right] .
$$

For larger magnetic fields, $N_{\phi}>1$, there are $N_{\phi}$ null lines in each of the $x$ and $y$ directions. This introduces a set of $N_{\phi}^{2}$ null points which form an evenly spaced square lattice (which may or may not coincide with the original lattice sites). These are indexed by $m, n=0, \ldots, N_{\phi}-1$

$$
\boldsymbol{R}_{0}^{m n}(\boldsymbol{\Theta})=\boldsymbol{R}_{0}(\boldsymbol{\Theta})+\frac{1}{N_{\phi}}\left(m L_{x}, n L_{y}\right),
$$

Correspondingly there are $N_{\phi}^{2}$ vorticity centers,

$$
\boldsymbol{R}_{\bigvee}^{m n}(\boldsymbol{\Theta})=\boldsymbol{R}_{0}^{m n}(\boldsymbol{\Theta})+\frac{1}{2}\left(L_{x}, L_{y}\right) .
$$

\section{A. Choosing a gauge}

The uniform magnetic field must integrate to an integer number of flux quanta $N_{\phi}$,

$$
B=\frac{2 \pi N_{\phi}}{q L_{x} L_{y}}
$$

The gauge field is given by

$$
\begin{gathered}
A_{\boldsymbol{r}, \boldsymbol{r}+\hat{\boldsymbol{x}}}^{x}=-\bmod \left(y-Y_{0}, L_{y}\right) B L_{x} H\left(X_{0}, x\right) \\
A_{\boldsymbol{r}, \boldsymbol{r}+\hat{y}}^{y}=\bmod \left(x-X_{0}, L_{x}\right) B .
\end{gathered}
$$

Note that for $0<m<n, \bmod (-m, n)=n-m$. The function $H\left(X_{0}, x\right)$ ensures that $A_{\boldsymbol{r}, \boldsymbol{r}+\hat{\boldsymbol{x}}}^{x}$ vanishes unless $\boldsymbol{r}$ is immediately to the left $(-\hat{x})$ of the null line $x=X_{0}$. It is defined by

$$
H\left(X_{0}, x\right)= \begin{cases}1 & 0<\bmod \left(X_{0}-x, L_{x}\right) \leq 1 \\ 0 & \text { otherwise }\end{cases}
$$

For a continuous position $\boldsymbol{r}$ we define $\boldsymbol{A}^{\alpha}(\boldsymbol{r})$ to be the linearly interpolated gauge field between the two enclosing links in the $\alpha$ direction.

For $\boldsymbol{\Theta}=(0,0)$ the null point $\boldsymbol{R}_{0}$ is at $\boldsymbol{R}_{0}=(0,0)$ and the vorticity center is therefore at $\boldsymbol{R}_{\mathrm{V}}=\left(\frac{1}{2} L_{x}, \frac{1}{2} L_{y}\right)$. Our gauge choice is shown in Fig. 3. The gauge invariant content of $\boldsymbol{A}$ consists of the uniform magnetic field with flux $B$ $=N_{\phi} \phi_{0} / L_{x} L_{y}$ in each plaquette, and the two Wilson loop functions 

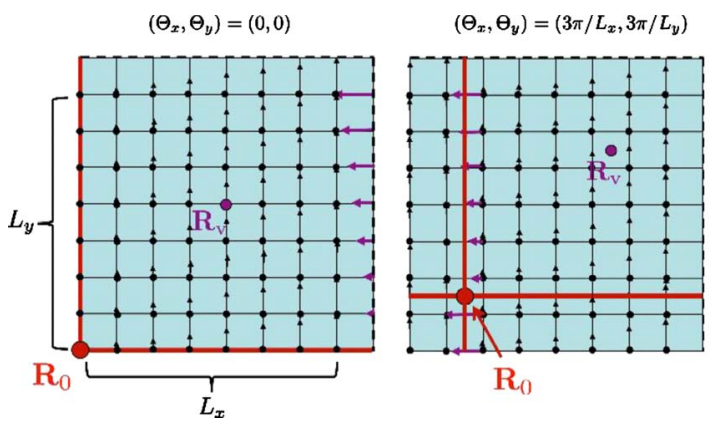

FIG. 3. (Color online) The lattice gauge field on links, $\boldsymbol{A}_{\boldsymbol{r}, \boldsymbol{r}+\boldsymbol{\eta}}$, according to Eq. (39), for two choices of $\mathrm{AB}$ parameters $\left(\Theta_{x}, \Theta_{y}\right)$. The length and thickness of the arrows are proportional to the magnitude of $\boldsymbol{A}$. For a single flux quantum, $N_{\phi}=1$, the two null lines are marked by red lines. The null points $\boldsymbol{R}_{0}(\boldsymbol{\Theta})$ and vorticity centers $\boldsymbol{R}_{\mathrm{V}}(\boldsymbol{\Theta})$ are depicted. Note that in the right figure, both points are located at plaquette centers.

$$
\begin{gathered}
W_{y}(x)=x q B L_{y}+\Theta_{y}, \\
W_{x}(y)=-y q B L_{x}+\Theta_{x} .
\end{gathered}
$$

\section{B. V-spin degeneracies}

In the process of calculating the Hall conductance (see Sec. VII), we computed the spectrum at half filling, for an even number of sites, with one total flux quantum of magnetic field. We encountered a sequence of $\mathrm{AB}$ fluxes $\boldsymbol{\Theta}_{i}$, where the whole spectrum becomes twofold degenerate. These degeneracy points are demonstrated in Fig. 4 for $N_{\phi}$ $=1$, for the lowest two multiplets. The level crossings indicate the existence of a noncommuting symmetry generators, ${ }^{31}$ which act on the wave functions of vortices introduced by the external magnetic field. We now construct these symmetry operators and compute their commutation relations.

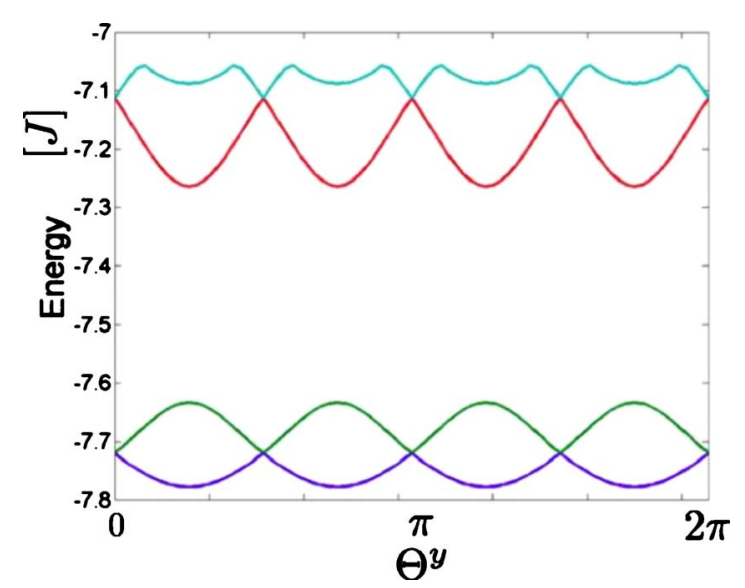

FIG. 4. (Color online) Emergence of "v-spin" degeneracies. Four lowest eigenenergies of $\mathrm{HCB}$ on the torus, with $N_{\phi}=1$, as a function of the $A B$ parameter $\Theta^{y}$, with $\Theta^{x}=0$. The spectrum separates into doublets. Notice the exact degeneracies which occur when the vorticity center coincides with a lattice position.
As discussed earlier, for a finite magnetic field $\left(N_{\phi}>0\right)$, $\mathcal{H}$ does not possess the lattice translational symmetry. Nevertheless, with respect to the vorticity center $\boldsymbol{R}_{\mathrm{V}}(\boldsymbol{\Theta})$ we can define two reflection operators

$$
\begin{aligned}
& P_{\mathrm{V}}^{x}(x, y)=\left[\bmod \left(2 X_{\mathrm{V}}-x, L_{x}\right), y\right], \\
& P_{\mathrm{V}}^{y}(x, y)=\left[x, \bmod \left(2 Y_{\mathrm{V}}-y, L_{y}\right)\right],
\end{aligned}
$$

which by Eq. (35) are equivalent to reflections about $\boldsymbol{R}_{0}$.

Now, by appropriately tuning $\Theta$ using Eq. (34), the vorticity center $\boldsymbol{R}_{\mathrm{V}}$ can be chosen to coincide with a symmetry point of the square lattice, such as any lattice site, bond center or plaquette center. Reflecting the Hamiltonian about that symmetry point, leads to

$$
\begin{aligned}
& P_{\mathrm{V}}^{\alpha} \mathcal{H}[\boldsymbol{A}] P_{\mathrm{V}}^{\alpha}=\mathcal{H}[\tilde{\boldsymbol{A}}], \\
& \tilde{\boldsymbol{A}}_{\boldsymbol{r}, \boldsymbol{r}+\boldsymbol{\eta}}^{\alpha}=\boldsymbol{A}_{P_{\mathrm{V}}^{\alpha}, P_{\mathrm{V}}^{\alpha}(\boldsymbol{r}+\boldsymbol{\eta})} .
\end{aligned}
$$

The gauge invariant content of $\widetilde{\boldsymbol{A}}^{\alpha}$ describes an inverted uniform magnetic field $\widetilde{B}=-B$, and a reversed sign of the Wilson loop functions [Eq. (32)].

The reversal of the fields in $\tilde{A}$ can be undone, at half filling, by applying the charge conjugation transformation $C$ [Eq. (5)], and a pure gauge transformation $U^{\alpha}$. Thus, we construct two operators,

$$
\begin{aligned}
& \Pi_{\mathrm{V}}^{x}=U^{x} C P_{\mathrm{V}}^{x}, \\
& \Pi_{\mathrm{V}}^{y}=U^{y} C P_{\mathrm{V}}^{y},
\end{aligned}
$$

where,

$$
U^{\alpha}=\exp \left[i \sum_{\boldsymbol{r}} \chi^{\alpha}(\boldsymbol{r}) S_{\boldsymbol{R}}^{z}\right]
$$

and

$$
\chi^{\alpha}(\boldsymbol{r})=\int_{\boldsymbol{R}_{0}}^{r} d \boldsymbol{r}^{\prime} \cdot\left[\boldsymbol{A}\left(\boldsymbol{r}^{\prime}\right)+\tilde{\boldsymbol{A}}^{\alpha}\left(\boldsymbol{r}^{\prime}\right)\right] .
$$

In the line integral we use the interpolated gauge field defined after Eq. (39). Since $\boldsymbol{A}$ and $-\widetilde{\boldsymbol{A}}^{\alpha}$ describe the same magnetic fields, they obey,

$$
\nabla \times\left(\boldsymbol{A}+\tilde{\boldsymbol{A}}^{\alpha}\right)=0 .
$$

This implies that $\chi^{\alpha}$ is independent of which continuous path (of zero winding number) is chosen between $\boldsymbol{R}_{0}$ and $\boldsymbol{r}$.

It is easy to verify by this construction that for all $\boldsymbol{\Theta}_{i}$ such that $\boldsymbol{R}_{\mathrm{V}}\left(\boldsymbol{\Theta}_{i}\right)$ is a symmetry point the $\Pi_{\mathrm{V}}^{\alpha}$ operators become symmetries of the Hamiltonian:

$$
\left[\mathcal{H}(\boldsymbol{A}), \Pi_{\mathrm{V}}^{\alpha}\right]=0, \quad \alpha=x, y .
$$

Now we calculate the commutation relation between $\Pi_{\vee}^{x}$ and $\Pi_{\mathrm{V}}^{y}$. This is a straightforward but slightly tedious procedure. Using the gauge choice [Eqs. (39) and (46)],

$$
\chi^{x}(\boldsymbol{r})=\frac{2 \pi N_{\phi}}{L_{y}} \bmod \left(y-Y_{0}, L_{y}\right)\left(1-\delta_{x, X_{0}}\right),
$$



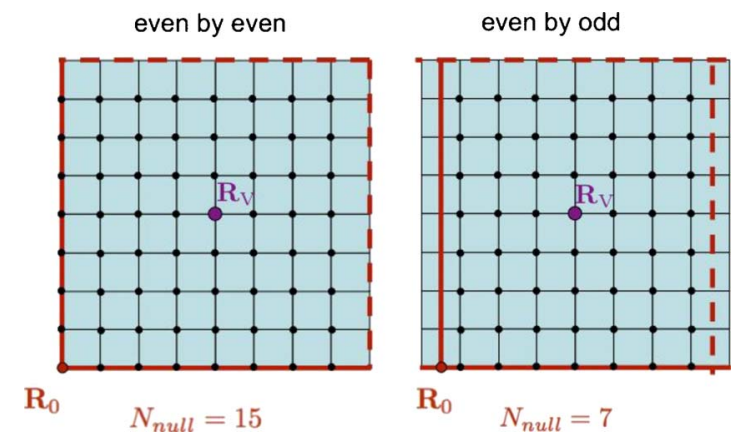

FIG. 5. (Color online) Figuring out $N_{\text {null }}$ for two cases of even size latices. The parity of the number of lattice sites (black dots) which lie precisely on the null lines (red color online), determines the commutation rule of $\Pi_{\mathrm{V}}^{x}$ and $\Pi_{\mathrm{V}}^{y}$, according to Eq. (53). The two examples explain why $N_{\text {null }}$ is odd whenever the vorticity center $\boldsymbol{R}_{\mathrm{V}}$ is positioned precisely on a lattice site.

$$
\chi^{y}(\boldsymbol{r})=0 .
$$

Note that $\chi_{\vee}^{x}$ vanishes on the null lines. Multiplying the two $\Pi_{\mathrm{V}}^{\alpha}$ operators yields

$$
\begin{gathered}
\Pi_{\mathrm{V}}^{y} \Pi_{\mathrm{V}}^{x}=\exp \left(i \sum_{\boldsymbol{r}}\left\{\chi^{y}-\chi^{x}\left[P_{\mathrm{V}}^{y}(\boldsymbol{r})\right]\right\} S_{\boldsymbol{r}}^{z}\right) P_{\mathrm{V}}^{y} P_{\mathrm{V}}^{x}, \\
\Pi_{\mathrm{V}}^{x} \Pi_{\mathrm{V}}^{y}=\exp \left(i \sum_{\boldsymbol{r}}\left\{\chi^{x}-\chi^{y}\left[P_{\mathrm{V}}^{x}(\boldsymbol{r})\right]\right\} S_{\boldsymbol{r}}^{z}\right) P_{\mathrm{V}}^{y} P_{\mathrm{V}}^{x}=e^{-i \mathrm{Y}} \Pi_{\mathrm{V}}^{y} \Pi_{\mathrm{V}}^{x},
\end{gathered}
$$

where we have used $\left[P_{\mathrm{V}}^{x}, P_{\mathrm{V}}^{y}\right]=0$. The overall phase is given by the operator

$$
\begin{gathered}
Y=\sum_{\boldsymbol{r}} \omega_{\boldsymbol{r}} S_{\boldsymbol{r}}^{z}, \\
\omega_{\boldsymbol{r}}=\chi^{x}+\chi^{x}\left(P_{\mathrm{V}}^{y}[\boldsymbol{r}]\right)-\chi^{y}-\chi^{y}\left(P_{\mathrm{V}}^{x}[\boldsymbol{r}]\right) .
\end{gathered}
$$

It can be directly verified from Eq. (49) that

$$
\omega_{r}= \begin{cases}0 & r \in \text { null lines } \\ 2 \pi N_{\phi} & \text { otherwise. }\end{cases}
$$

Since $\exp \left(i 2 \pi m S_{r}^{z}\right)=(-1)^{m}$,

$$
e^{-i \Upsilon}=(-1)^{N_{\phi}\left(N-N_{\text {null }}\right)}=(-1)^{N_{\phi} N_{\text {null }},}
$$

where $N_{\text {null }}$ is the number of sites which sit precisely on the two null lines. For even $N_{\phi}, e^{i \Upsilon}=1$ and $\left[\Pi_{\mathrm{V}}^{x}, \Pi_{\mathrm{V}}^{y}\right]=0$.

For odd $N_{\phi}$, and odd $N_{\text {null }}$, one obtains $e^{-i \Upsilon}=-1$. Let us prove a simple lemma concerning the parity of $N_{\text {null }}$.

Lemma. For even size lattices, if $\boldsymbol{R}_{\mathrm{V}}$ is tuned to be precisely on a lattice site, then $N_{\text {null }}$ is odd. The proof is illustrated in Fig. 5.

Proof. Since we assume that $N$ is even (to describe precise half filling), there are two cases to consider: (i) for an even by even lattice, $\boldsymbol{L}=(2 m, 2 n)$, if we choose $\boldsymbol{R}_{0}$ on a lattice site, it is easy to see that $\boldsymbol{R}_{\bigvee}$ must also sit on a lattice site. The number of sites which contribute to $N_{\text {null }}$ are the sum of lattice sites in the $x$ and $y$ directions minus the null point itself which is counted twice

$$
N_{\text {null }}^{\mathrm{ee}}=2 m+2 n-1 .
$$

Hence $e^{i \Upsilon}=-1$. (ii) For the odd by even lattice, e.g., $\boldsymbol{L}$ $=(2 m+1,2 n)$, we choose $\boldsymbol{R}_{0}$, to be in the middle of a bond in the $x$ direction. The null line includes only the sites on the $x$-null line which is odd

$$
N_{\text {null }}^{\mathrm{eo}}=2 m+1
$$

Thus, here too $e^{-i \Upsilon}=-1$. Note that in both Eqs. (54) and (55), the vorticity center is situated on lattice sites $\boldsymbol{R}_{\mathrm{V}}=(m, n)$. QED.

Thus we conclude that for an odd number of fluxes $N_{\phi}$ $=2 n+1$, if $\boldsymbol{R}_{\mathrm{V}}\left(\boldsymbol{\Theta}_{i}\right)$ is located precisely on any lattice site, then $\Pi_{\mathrm{V}}^{x}$ and $\Pi_{\mathrm{V}}^{y}$ anticommute.

Under these conditions, all states of $\mathcal{H}\left[\boldsymbol{\Theta}_{i}\right]$ must be at least twofold degenerate. This follows the standard proof: Since

$$
\left[\mathcal{H}, \Pi_{\mathrm{V}}^{x}\right]=0,
$$

and $\Pi_{V}^{x}$ has eigenvalues \pm 1 , then each common eigenstate of $\mathcal{H}$ and $\Pi_{\mathrm{V}}^{x}$, can be labeled by $\left|E_{n}, \pi^{x}= \pm 1\right\rangle$. Now,

$$
\Pi_{\bigvee}^{x} \Pi_{\bigvee}^{y}\left|E_{n}, 1\right\rangle=-\Pi_{\bigvee}^{y} \Pi_{\bigvee}^{x}\left|E_{n}, 1\right\rangle \propto\left|E_{n},-1\right\rangle,
$$

that is to say each eigenenergy $E_{n}$ is associated with a degenerate pair of eigenstates with opposite quantum numbers of $\Pi_{\mathrm{V}}^{x}$.

$\Pi_{\vee}^{x}$ and $\Pi_{\bigvee}^{y}$ are point group symmetries about the vorticity center. We can also construct a third symmetry operator $\Pi^{z}$ as

$$
\Pi_{\mathrm{V}}^{z}=-i \Pi_{\mathrm{V}}^{x} \Pi_{\mathrm{V}}^{y} .
$$

The three operators $\Pi_{\mathrm{V}}^{\alpha}=\left(\Pi_{\mathrm{V}}^{x}, \Pi_{\mathrm{V}}^{y}, \Pi_{\mathrm{V}}^{z}\right)$ are unitary and Hermitian,

$$
\Pi_{\mathrm{V}}^{\alpha}=\left(\Pi_{\mathrm{V}}^{\alpha}\right)^{\dagger}=\left(\Pi_{\mathrm{V}}^{\alpha}\right)^{-1} \Rightarrow\left(\Pi_{\mathrm{V}}^{\alpha}\right)^{2}=1 .
$$

Therefore their eigenvalues are \pm 1 . The $\Pi$ operators behave as Pauli matrices and can be used to construct an SU(2) algebra of spin half,

$$
\tau^{\alpha}=\frac{1}{2} \Pi_{\mathrm{V}}^{\alpha}, \quad \alpha=x, y, z .
$$

We note that for multiple number of magnetic fluxes $N_{\phi}$ $>1$, degeneracies appear for any odd number of vortices. This is consistent with the Kramers doublets associated with an odd number of interacting spin half particles.

\section{C. v-spin and meron density}

The semiclassical analysis of HCB vortices at half filling, shown in Eq. (28) finds that the vortex has a CDW in its core. This is signaled by the local order parameter $\left\langle n^{z}(\boldsymbol{r})\right\rangle$ $\neq 0$, as illustrated in Fig. 1 .

We define the modified "meron density" operator as

$$
\begin{gathered}
\tau^{z}(\boldsymbol{r}) \simeq \hat{\boldsymbol{n}} \cdot D_{x} \hat{\boldsymbol{n}} \times D_{y} \hat{\boldsymbol{n}}, \\
D_{\alpha} \equiv \partial_{\alpha}-i q \boldsymbol{A}_{\alpha},
\end{gathered}
$$

where $\hat{\boldsymbol{n}}$ were defined in Eq. (21) and $D_{\alpha}$ are gauge invariant derivatives in the $x y$ plane. In the absence of a gauge field 
$\boldsymbol{A}=0$, a single meron (half a skyrmion) of a continuos classical field has topological charge

$$
Q=\frac{1}{4 \pi} \int d^{2} r \tau^{z}(\boldsymbol{r}, \boldsymbol{A}=0)= \pm \frac{1}{2} .
$$

An HCB representation of $\tau_{r}^{z}$ is constructed using spin-half operators. In the presence of one flux quantum, we define the modified topological charge operator as

$$
\widetilde{Q}=\frac{1}{4 \pi} \sum_{r} \tau_{r}^{z}(\boldsymbol{A}) .
$$

$\widetilde{Q}$ is not expected to be quantized at $\pm \frac{1}{2}$. However, we have found that in the low-lying eigenstates of Eq. (4), its sign correlates with the eigenvalues of $\Pi^{z}$ :

$$
\operatorname{sign}\left(\left\langle E_{n}, \pi^{z}|Q| E_{n}, \pi^{z}\right\rangle\right)=\pi^{z} .
$$

We conclude that $\pi^{z}$ of a single vortex measures the sign of the CDW in its core, with respect to sublattice A.

$C D W$ near vortex cores. Density modulations have been observed near vortex cores of high- $T_{\mathrm{c}}$ cuprate superconductors ${ }^{32}$ and analyzed within bosonic models. Previous theoretical work explained the $\mathrm{CDW}$ correlations variationally, ${ }^{27}$ or as a result of Berry phase ${ }^{33}$ which appear in the effective hamiltonian Eq. (29). The Berry phases can be used as part of a semiclassical argument to show that the effective single vortex wave functions are twofold degenerate, in the infinite lattice limit. The operator construction of $\mathrm{v}$-spins, however, allows us to identify the mathematical reason for the exact doublet degeneracies in the many body spectrum on any finite torus, with any odd number of vortices. The v-spins can thus be used to describe also the excitations and dynamical correlations for the multiple vortex system, both in the vortex solid and the vortex liquid phase.

\section{HAMILTONIAN OF QUANTUM VORTICES}

\section{A. Vortex confining potential}

The current density operator is given in the pseudospin representation by

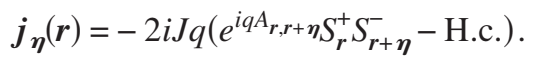

By choosing $\boldsymbol{A}$ to describe one flux quantum of uniform magnetic field through the whole lattice one vortex is introduced into the low-energy eigenstates. Indeed, we verified that the exact ground state exhibits a vortex pattern of the current density $\left\langle j_{\eta}\right\rangle$, defined in Eq. (65). Also, the center of vorticity is agrees with the value of $\boldsymbol{R}_{\mathrm{V}}(\boldsymbol{\Theta})$ as defined in Eqs. (34) and (35).

We determine the effective confining potential on the vortex variationally. We choose the square geometry $L^{2}=N$, and define a vortex coherent state centered at $\boldsymbol{R}$ by a spin coherent state $\left|\hat{\boldsymbol{\Omega}}_{\mathrm{V}}[\boldsymbol{R}]\right\rangle$. All the unit vectors $\hat{\boldsymbol{\Omega}}_{\mathrm{V}}$ lie in the $x y$ plane with the azimuthal angles $\phi_{r}$ given by a Jacobi theta function $^{34}$

$$
\phi_{\boldsymbol{r}}(\boldsymbol{R})=-\operatorname{Im} \log \vartheta\left[i(z-Z)-\frac{1}{2}-\frac{i}{2}\right]+\pi\left[y-\left(\frac{1}{2}-Y\right) x\right],
$$

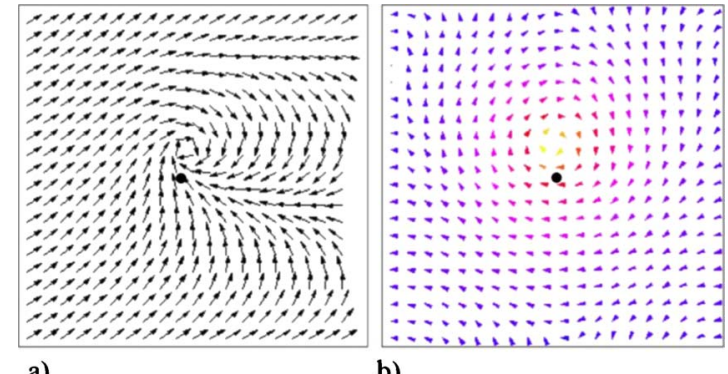

a)

b)

FIG. 6. (Color online) A variational vortex configuration with its center located at $\boldsymbol{R}=(0.5,0.6) \boldsymbol{L}$. The black dots mark the center of vorticity $\boldsymbol{R}_{\mathrm{V}}$, where the variational energy is minimized. (a) Phase field marked by directions of the arrows. (b) Current density distribution where the colors correspond to the local current magnitude. Note the excess currents flowing around the torus in the negative $x$ direction: a consequence of the vortex being displaced from variational minimum.

$$
\vartheta(z)=\sum_{n=-\infty}^{\infty} e^{-\pi n^{2}} e^{2 i \pi n z} .
$$

Here we use scaled complex coordinates $z=(x+i y) / L$ and $Z=(X+i Y) / L$ for the position and vortex center $\boldsymbol{r}$ and $\boldsymbol{R}$, respectively.

While $\phi_{r}$ is discontinuous on the torus at $x, y=L$, the gauge invariant current density

$$
\left\langle\hat{\boldsymbol{\Omega}}_{\mathrm{V}}\left|j_{\boldsymbol{\eta}}\right| \hat{\boldsymbol{\Omega}}_{\mathrm{V}}\right\rangle=q J \sin \left(\phi_{\boldsymbol{r}}-\phi_{\boldsymbol{r}+\boldsymbol{\eta}}-\boldsymbol{A}_{\boldsymbol{r}, \boldsymbol{r}+\boldsymbol{\eta}}\right),
$$

is continuous. An example for the phase and current distributions is given in Fig. 6 .

The effective confining potential on the vortex is given by the classical energy defined in Eq. (10). By semiclassical estimates ${ }^{35}$ its curvature at $\boldsymbol{R}_{\mathrm{V}}$ scales as $1 / N$. For lattices of size $L \geq 4$ we fit the variational potential by a twodimensional (2D) quadratic function, which scales as $1 / N$ as,

$$
U_{N}=\frac{1}{2} K\left|\boldsymbol{R}-\boldsymbol{R}_{\mathrm{V}}\right|^{2} / N, \quad K=39.2 J .
$$

$U$ is minimized at the vorticity center $\boldsymbol{R}_{\mathrm{V}}$, which was defined in Eq. (35). Figure 7 depicts the confining potential as a function of vortex center for the choice of $\boldsymbol{\Theta}=(0,0)$.

We can now combine the single vortex hopping terms of Eq. (29) with the confining potential to obtain the Harper hamiltonian on the finite torus

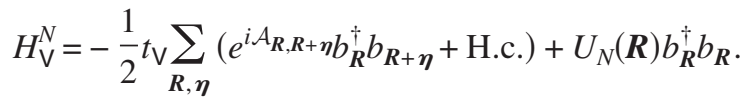

\section{B. Vortex-hopping amplitude}

For a quantitative quantum theory of vortices we need to evaluate the effective hopping $t_{\mathrm{V}}$. Since vortex tunneling between lattice sites depends on short range many-body correlations, we extract $t_{\mathrm{V}}$ from exact numerical diagonalizations of $\mathcal{H}$ on 16-20 sites clusters, in the presence of a single flux quantum. 


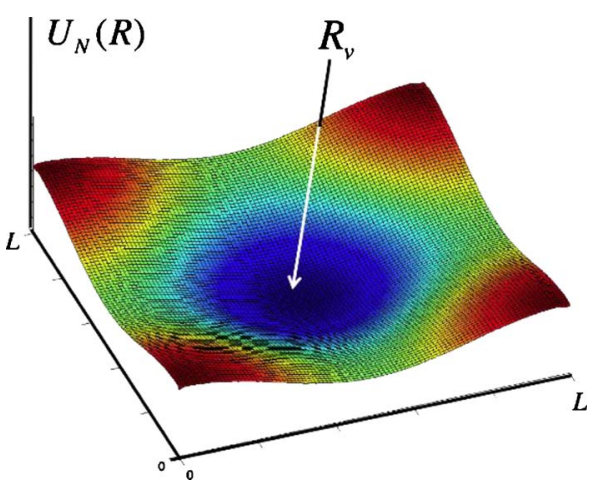

FIG. 7. (Color online) The energy as a function of vortex position, for a single vortex on the torus of dimensions $L \times L$. The vorticity center is located at $\boldsymbol{R}_{\mathrm{V}}=\frac{1}{2} \boldsymbol{L}$.

By tuning $t_{\mathrm{V}}$, we fit the lowest three eigenenergies $E_{n}$ of $\mathcal{H}$ to those of the effective Harper Hamiltonian (70). The fit is shown in Fig. 8.

Our primary concern is that the low eigenstates will not be exclusively described by $H_{\mathrm{V}}^{N}$ since there are also lowenergy superfluid phonons (phase fluctuations). ${ }^{36-38}$ However, we can estimate the phonons lowest excitation to be gapped by the finite lattice with the energy scale $2 \pi J / L$, which is larger than the energies we have fitted to Eq. (70).

Our results for $t_{\mathrm{V}}\left(n_{\mathrm{b}}, V / J\right)$, for $N=20$ can be described by the fitting formulas,

$$
t_{\mathrm{V}}(n, 0)=4 J-50.4 J\left(n-\frac{1}{2}\right)^{2}+5056 J\left(n-\frac{1}{2}\right)^{4},
$$

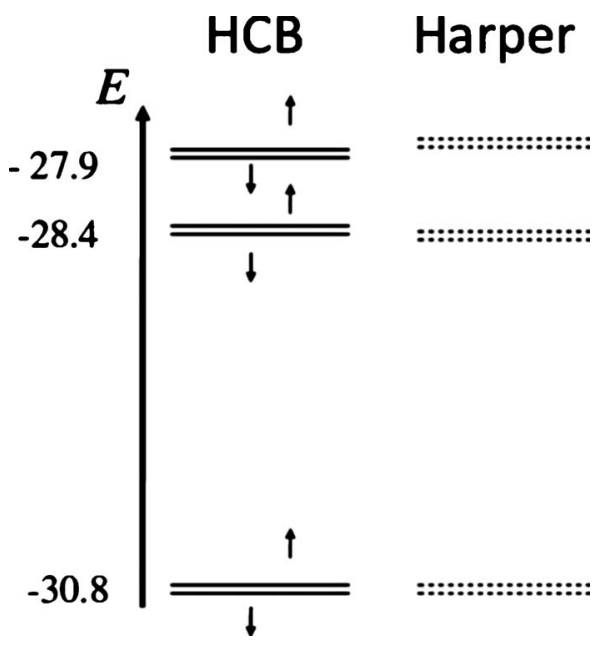

FIG. 8. Fitting the single vortex energies of the effective Harper Hamiltonian (70), to the many-body spectrum of HCB [Eq. (4)]. The HCB Hamiltonian is defined on a $4 \times 4$ lattice at half filling, which is embedded on a torus which is penetrated by one flux quantum. The parameters $J=1$, and $V=0$ are chosen for this figure. $\mathrm{Up}$ and down arrows denote the $\mathrm{v}$-spin magnetic quantum number $\tau^{z}$. The confining potential of the vortex in the Harper Hamiltonian is calculated by a variational calculation, Eq. (69). The effective vortex hopping rate is fit to be $t_{\mathrm{V}}=4 \mathrm{~J}$. The lowest three doublets of the two models agree within $2 \%$ of the first energy gap.

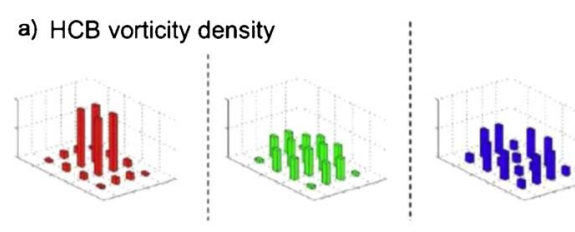

b) Vortex wavefunctions probability density

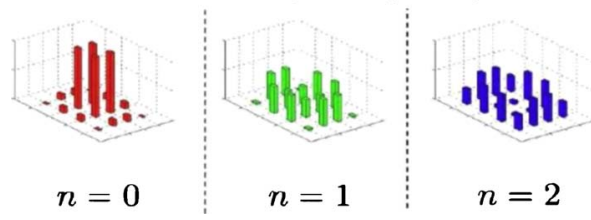

FIG. 9. (Color online) (a) The HCB vortices density $\rho_{\mathrm{v}}^{(n)}(\boldsymbol{R})$ as defined in Eq. (72)), for the states $|n, \uparrow\rangle$ whose spectrum is depicted in Fig. 8. A uniform background vorticity has been subtracted. (b) Probability density of the lowest single particle states of the Harper model given by Eq. (70). The qualitative similarity between (a) and (b) supports the fit of the vortex hopping rate, and the validity of the Harper Hamiltonian for single vortex dynamics.

$$
t_{\mathrm{V}}\left(\frac{1}{2}, V\right)=4 J+6 V+10.8 \frac{V^{2}}{J} .
$$

The system parameters were varied throughout the range $\mid n$ $-\frac{1}{2} \mid \leq 0.2$, and $V / J<0.5$. We find that at half filling, the vortex hopping rate $t_{\mathrm{V}}$ varies very little between the $N=16$ and $N=20$ lattices. This indicates that the bare vortex kinetic energy is determined by short range correlations, and thus does not require a large lattice to be computed with acceptable accuracy.

To further test the association of the many-body lowest eigenstates with vortex center fluctuations, we measure the vorticity density defined on the dual lattice,

$$
\rho_{\mathrm{v}}^{(n)}(\boldsymbol{R}) \equiv \sum_{\boldsymbol{r}, \boldsymbol{\eta}}^{\text {plaq, } \boldsymbol{R}}\left\langle\Psi_{n}\left|\boldsymbol{j}_{\boldsymbol{r}, \boldsymbol{\eta}}\right| \Psi_{n}\right\rangle \cdot \boldsymbol{\eta},
$$

for each of the low-lying states $\left|\Psi_{n}\right\rangle$. We compare $\rho_{\mathrm{V}}$ to the single particle probability density of the corresponding wave functions of $H_{\mathrm{V}}^{N}$. As shown in Fig. 9, for the fitted value of $t_{\mathrm{V}}$, the corresponding distributions increase in width in a qualitatively similar fashion. This demonstrates that the low-lying eigenstates of $\mathcal{H}$ correspond to quantum fluctuations of the vortex position.

\section{QUANTUM MELTING OF THE VORTEX LATTICE}

At half filling, for $N_{\phi}>1$ semiclassical evaluation of vortex interactions at distances larger than the core radius is

$$
U_{i j}^{\mathrm{int}}=-\pi J \log \left(\left|\boldsymbol{R}_{i}-\boldsymbol{R}_{j}\right|\right) .
$$

Integrating out the phonon fluctuations, produces an instantaneous logarithmic (2D Coulomb) interaction between vortices, plus retarded (frequency-dependent) interactions. ${ }^{25,37}$ Since we are interested in the short wavelength fluctuations which are responsible for quantum melting of the vortex lattice, we ignore these retardation effects. 
Thus in the large lattice limit, at a finite magnetic field $B$, the multivortex quantum hamiltonian is given by the Harper boson plasma

$$
\begin{aligned}
& H_{\mathrm{HBP}}=-\frac{1}{2} t_{\mathrm{V}} \sum_{\langle i j\rangle}\left(e^{i \mathcal{A}_{i j}} b_{i}^{\dagger} b_{j}+\text { H.c. }\right)-\pi J \sum_{i, j} n_{i} n_{j} \log \left(\left|\boldsymbol{R}_{i}-\boldsymbol{R}_{j}\right|\right) \\
& +\pi^{2} J \frac{B}{\Phi_{0}} \sum_{i} n_{i}\left|\boldsymbol{R}_{i}\right|^{2} .
\end{aligned}
$$

At half filling the continuum limit of Eq. (75) can be taken as follows. The single vortex dispersion exhibits a twofold degeneracy of the ground states of the Harper Hamiltonian at $\pi$ flux per plaquette. This implies the degeneracy $E_{k}=E_{k+(0, \pi)}$, at low $|\boldsymbol{k}| \ll \pi$. Since we wish to expand the hamiltonian at long wavelength, we retain the degeneracy by the v-spin label $s=\uparrow, \downarrow$. The vortex effective mass is defined as

$$
M_{\mathrm{V}}=\left(\frac{\partial^{2} E_{\boldsymbol{k}}}{\partial \boldsymbol{k}^{2}}\right)^{-1}=1 / t_{\mathrm{V}}
$$

This leads to the continuum spin-half Coulomb Bosons (CB) Hamiltonian for the vortices at half filling:

$$
\mathcal{H}_{\mathrm{CB}}=\sum_{i, s=\uparrow \downarrow} \frac{\boldsymbol{P}_{i}^{2}}{2 M_{\mathrm{V}}}+\pi J \sum_{i \neq j} \log \left(\left|\boldsymbol{R}_{i}-\boldsymbol{R}_{j}\right|\right)-\frac{B}{\Phi_{0}} \pi^{2} J \sum_{i}\left|\boldsymbol{R}_{i}\right|^{2} .
$$

At low-vortex densities, interactions clearly must dominate over the kinetic energy, and vortices form a vortex lattice. This a superfluid phase with v-spin correlations.

At a finite temperature $T_{m}$, if we ignore the quantum effects of the kinetic term, the classical melting temperature $T_{m}$ is independent of vortex density (magnetic field). ${ }^{39,40}$ (Any change in $B$ can be absorbed by scaling $\boldsymbol{R}_{i}$ appropriately, leaving the classical energy invariant).

However, quantum fluctuations of the Coulomb plasma Eq. (76), increase with the vortex density $B / \Phi_{0}$, until quantum melting is reached at a critical vortex density $B_{\mathrm{cr}}$. This melting is analogous to that of spinless CB, studied by Magro and Ceperley (MC) (Ref. 41) by diffusion Monte Carlo.

$\mathrm{MC}$ used the dimensionless parameter to describe the $\mathrm{CB}$ density $n_{\mathrm{v}}$,

$$
r_{s}^{-2}=\pi n_{\mathrm{v}} a_{0}^{2},
$$

where $a_{0}$ is the Bohr radius. We set the Bohr radius to be

$$
a_{0}=\left(\frac{\hbar^{2}}{\pi J M_{\mathrm{V}}}\right)^{1 / 2}
$$

to match between the model of $\mathrm{MC}$ and our $\mathcal{H}_{\mathrm{CB}}$ of Eq. (76).

$\mathrm{MC}$ found that below $r_{s} \approx 12$ the boson lattice undergoes quantum melting, i.e., they found a critical quantum melting density of

$$
n_{\mathrm{v}}^{\mathrm{cr}}=\frac{1}{144 \pi a_{0}^{2}} .
$$

Above this density, the $\mathrm{CB}$ looses translational symmetry breaking and becomes a quantum liquid, which will be discussed in Sec. VIII. Using our values of $M_{\mathrm{v}}$ from Eqs. (71) and (75), this translates into a critical vortex number per lattice site of

$$
n_{\mathrm{v}}^{c r} \leq\left(6.5-7.9 \frac{\mathrm{V}}{\mathrm{J}}\right) 10^{-3} \text { vortices per site. }
$$

This is a surprisingly low-vortex density, above which a vortex quantum liquid (QVL) is created. We shall briefly return to discuss the QVL in Sec. VIII

\section{HALL CONDUCTIVITY}

We have shown earlier by Eqs. (7) and (20) that the Hall conductivity in the low and high density obey the effective Galilean invariant limits,

$$
\sigma_{x y}= \begin{cases}\frac{n q}{B} & n \ll \frac{1}{2} \\ -\frac{(1-n) q}{B} & 1-n \ll \frac{1}{2} .\end{cases}
$$

In terms of vortex motion, this relation implies that below and above half-filling vortices drift in opposite directions relative to the particle current. In the following we shall study the transition between these two regimes. Since the continuum approximation is expected to fail near half filling, we resort to a numerical computation of $\sigma_{x y}$.

The zero-temperature Hall conductance of a finite lattice embedded on a torus is defined by the Chern number ${ }^{42}$

$$
\sigma_{x y}=\frac{q^{2}}{h \pi} \int_{0}^{2 \pi} d \Theta_{x} \int_{0}^{2 \pi} d \Theta_{y}\left\langle\frac{\partial \Psi_{0}}{\partial \Theta_{x}} \mid \frac{\partial \Psi_{0}}{\partial \Theta_{y}}\right\rangle,
$$

where $\left|\Psi_{0}(\boldsymbol{\Theta})\right\rangle$ is the exact ground state of Eq. (4), in the presence of $\mathrm{AB}$ fluxes $\boldsymbol{\Theta} . q^{2} / h$ is the quantum of conductance. In the absence of degeneracies and level crossings, the Chern number $\sigma_{x y} h / q^{2}$ is an integer. We compute Eq. (82) for a sequence of finite lattices. In Fig. 10 we plot $\sigma_{x y}$ for a square lattice of size $4 \times 4$ with a single flux quantum $N_{\phi}$ $=1$, as function of boson numbers $N_{b}=[0,1, \ldots, 16]$. We find that the Hall conductance follows two straight lines as given by Eq. (81), with an abrupt jump to zero at half filling. The same behavior was found for all smaller lattices, and reflects a sharp change in vortex dynamics around half filling. By Eq. (7), the Hall conductivity (and therefore the vortex Magnus action) much vanish at half filling by charge conjugation symmetry.

We extend Eq. (82) to finite temperatures by thermally averaging over all eigenstates $\left|\Psi_{n}\right\rangle$,

$$
\sigma_{x y}(T)=\frac{q^{2}}{h \pi} \sum_{n=0}^{\infty} \int_{0}^{2 \pi} d \Theta_{x} \int_{0}^{2 \pi} d \Theta_{y} \frac{e^{-E_{n} / T}}{Z} \operatorname{Im}\left\langle\frac{\partial \Psi_{n}}{\partial \Theta_{x}} \mid \frac{\partial \Psi_{n}}{\partial \Theta_{y}}\right\rangle .
$$

$E_{n}(\boldsymbol{\Theta})$ and $\left|\Psi_{n}(\boldsymbol{\Theta})\right\rangle$ are the exact spectrum and eigenstates of Eq. (4). The results obtained with Eq. (83) are matched at high temperatures with the conductivity calculated using the Lehmann representation of the Kubo formula, ${ }^{43}$ 


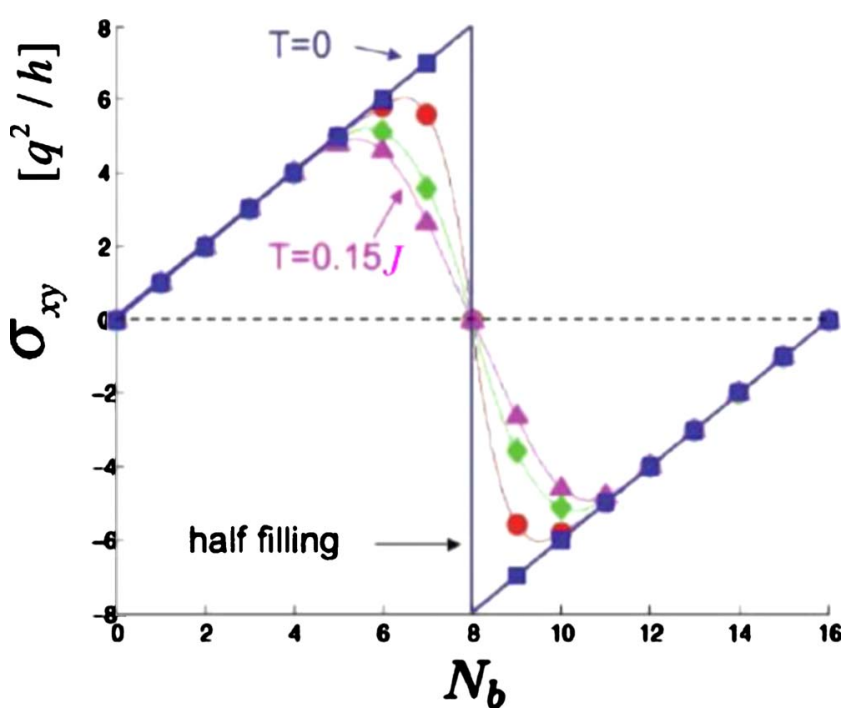

FIG. 10. (Color online) Hall conductance as a function of boson number $N_{b}$ for hard-core bosons on a $4 \times 4$ lattice on the torus with one penetrating flux quantum. Temperatures vary in intervals of $\Delta T=0.05 \mathrm{~J}$. The jump of the zero temperature conductance at half filling, is smoothened at finite temperatures.

$$
\begin{aligned}
\sigma_{x y}(T)= & \lim _{\omega \rightarrow i 0^{+}} \frac{i}{N Z \omega} \sum_{m, n} \frac{e^{-\beta E_{m}}-e^{-\beta E_{n}}}{E_{n}-E_{m}+\omega}\left\langle\Psi_{n}\left|\sum_{r} j_{x}(\boldsymbol{r})\right| \Psi_{m}\right\rangle \\
& \times\left\langle\Psi_{m}\left|\sum_{\boldsymbol{r}^{\prime}} j_{y}\left(\boldsymbol{r}^{\prime}\right)\right| \Psi_{n}\right\rangle,
\end{aligned}
$$

where the current operator $\boldsymbol{j}_{\boldsymbol{\eta}}$ is defined by Eq. (65).

The Kubo expression for $\sigma_{x y}$ is evaluated at high enough temperatures where the $\omega \rightarrow 0$ limit is well behaved. In Fig. 11 we plot $\sigma_{x y}$ as a function of temperature at different HCB densities, by interpolating between Eq. (83) at low temperatures and Eq. (84) at high temperatures.

We see that in general, the magnitude of $\sigma_{x y}\left(T, n_{\mathrm{b}}\right)$ decreases with temperature, and the discontinuity as a function of filling at zero temperature smoothens at finite temperatures. As the temperature is lowered, the reversal of the Hall conductance takes place in a narrower region around half filling. A characteristic Hall temperature $T_{H}\left(n_{\mathrm{b}}\right)$ can be defined by,

$$
\sigma_{x y}\left(T_{H}\right)=\frac{1}{2} \sigma_{x y}(0)
$$

In the inset of Fig. 10, we show that $T_{\mathrm{H}}$ increases with $\mid n_{\mathrm{b}}$ $-\frac{1}{2} \mid$, although we cannot estimate the critical exponent from the small cluster calculation.

\section{DISCUSSION AND EXPERIMENTS}

In this paper, we have determined the vortex effective hopping rate $t_{\mathrm{V}}$, in Eq. (71), and the Hall conductivity $\sigma_{x y}$, in Fig. 10, from the eigenstates of 16 site clusters on the torus. We emphasize that these quantities serve only as short wavelength "bare parameters," to be used to in the single vortex Harper Hamiltonian Eq. (75) and the multivortex Bose Cou-

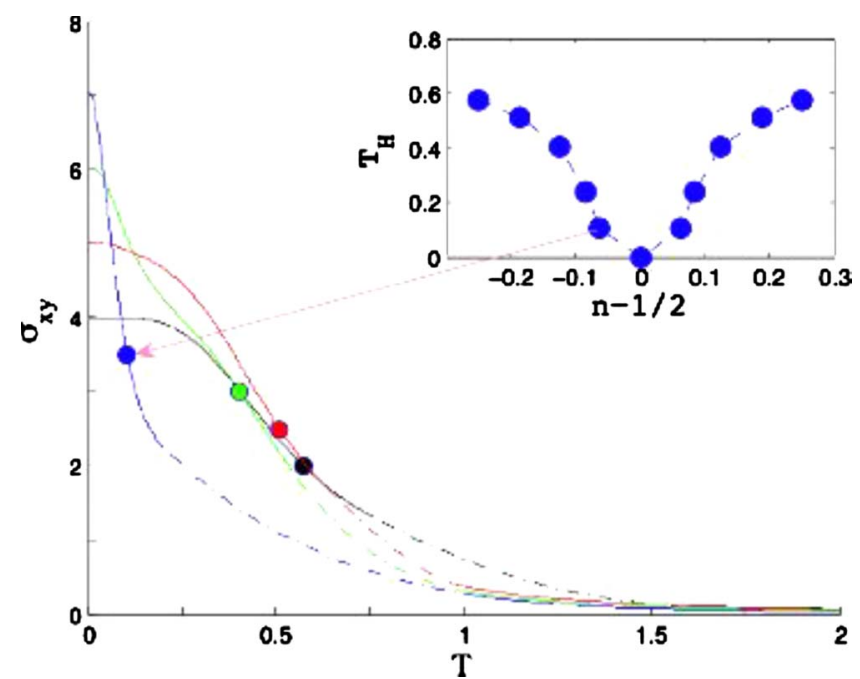

FIG. 11. (Color online) $\sigma_{x y}$ of hard-core bosons, as a function of temperature (in units of $t_{\mathrm{V}}$ ), for $4-7$ bosons on a $4 \times 4$ lattice. For low temperatures, we calculate $\sigma_{x y}$ using Eq. (83) while for $T>1$ we use Eq. (84). The dashed line is an interpolation between the two calculations. The points where $\sigma_{x y}$ drops to half its value at $T$ $=0$ are indicated. The inset shows the temperature scale $T_{H}$ as a function of density difference from half filling.

lomb liquid model Eq. (75). The charge transport coefficients depend on the thermodynamic phases of the latter model.

Vortex Solid. The vortex solid phase, expected for vortex densities lower than $n_{\mathrm{v}}^{c r}$ of Eq. (80), has superfluid (i.e., superconducting) order. Vortices are pinned to their lattice positions and therefore $\sigma_{x x}=\infty$ and $\sigma_{x y}=0$. The role of $t_{\mathrm{V}}$ is to produce quantum zero point motion and effective $\mathrm{v}$-spin super-exchange interactions, which are ferromagnetic (since vortices have Bose statistics). Below the $\mathrm{v}$-spin ordering temperature $T_{\text {vspin }}$, charge density waves might be expected with significant magnitude in the vortex cores. This phase is a weak supersolid. As mentioned earlier, density modulations have been observed near vortex cores of high- $T_{\mathrm{c}}$ cuprate superconductors. ${ }^{32}$ We note that within the HCB model, the interactions between $\mathrm{V}$-spins are expected to decay rapidly at low-vortex densities since vortex cores are well localized. This implies low values of $T_{\text {vspin }}$. At $T>T_{\text {vspin }}$ the v-spins disorder, and will contribute to the entropy density a term linear in magnetic field,

$$
s_{\mathrm{vspin}}=\frac{B}{\Phi_{0}} \log 2 .
$$

Quantum vortex liquid. At vortex densities which exceed $6.5 \times 10^{-3}$ vortices per lattice site, we expect the vortex lattice to melt and give way to the Boson Coulomb liquid studied by MC. ${ }^{41}$ Superfluidity of the CB translates into a Mott insulating behavior of the original bosons. ${ }^{36}$ However, MC have found that the liquid phase Eq. (76) is incompressible and hence exhibits vanishing condensate fraction. ${ }^{44}$ Furthermore, retardation effects act to suppress dual superfluidity. ${ }^{45}$ The value of the transport coefficients of the QVL phase is therefore left as an important open question. Away from half filling, our results for $\sigma_{x y}$ show that the vortices are subject 


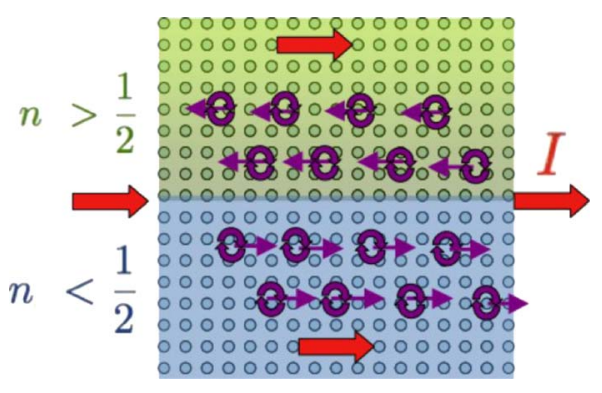

FIG. 12. (Color online) Different vortex drift directions (online purple arrows) in the presence of a bias current (online red arrows), for regions of boson density $n$ which is lower (blue online) and higher (green online) than half filling.

to a strong magnetic field, which further suppresses their condensation. At low boson fillings and large vortex density, $n_{\mathrm{b}} / n_{\phi}<1$, there is evidence for fractional quantum hall phases. ${ }^{46,47}$

The QVL phase discussed above is distinct from the vortex-antivortex condensate phases which were predicted at rational boson filling fractions, $n_{\mathrm{b}}=p / q$, in the absence of a magnetic field. ${ }^{33,48-50}$ These are expected at strong longer range interactions $V \approx J$, and corresponds to Mott-insulating commensurate $\mathrm{CDW}$ phases.

Hall coefficient. The abrupt reversal of Hall coefficient was found for 16 and 20 site lattices. This effect correlates with the rapid change in the semiclassical vortex core profile at half filling since we know by the GP Eq. (17) that vortices have a diverging density depletion (accumulation) in the low- and (high-) filling regime while they have a localized charge density wave in their small core at half filling. The sign of $\sigma_{x y}$ determines the drift direction of a vortex with respect to a bias current. This rapid reversal of $\sigma_{x y}$ may be relevant to the rapid change in Hall resistivity as a function of doping, which was observed in $\left(\mathrm{La}_{1-x} \mathrm{Sr}_{x}\right)_{2} \mathrm{CuO}_{4} \cdot{ }^{51,52}$

In Fig. 12 we propose a setup to observe the Magnus action reversal for cold bosonic atoms on a rotating optical trap. ${ }^{53}$ If the density of bosons is allowed to vary slowly in space across half filling, we expect a rapid change in the vortex drift directions at the half-filling line. The vortices would drift downstream with the boson current for $n<\frac{1}{2}$ and upstream for $n>\frac{1}{2}$.

Soft core interactions. We have not considered relaxing the hard-core constraints of the bosons, which would be described by the Bose Hubbard model [Eq. (1)]. For $U / J<\infty$, the charge conjugation operator ceases to be an exact symmetry at half filling, and Eq. (6) is not valid. Therefore the Hall coefficient will not be precisely zero at half filling, and the $\mathrm{v}$-spin degeneracies will be lifted by the finite $U / J$ corrections. A full determination of the Hall conductivity in the $U / J$ versus $n$ phase diagram, is an interesting open question. In particular, one would like to find out how the zeros of the Hall conductivity connect between the HCB limit and the free bosons limit.

\section{ACKNOWLEDGMENTS}

We thank Ehud Altman, Yosi Avron, David Ceperley,

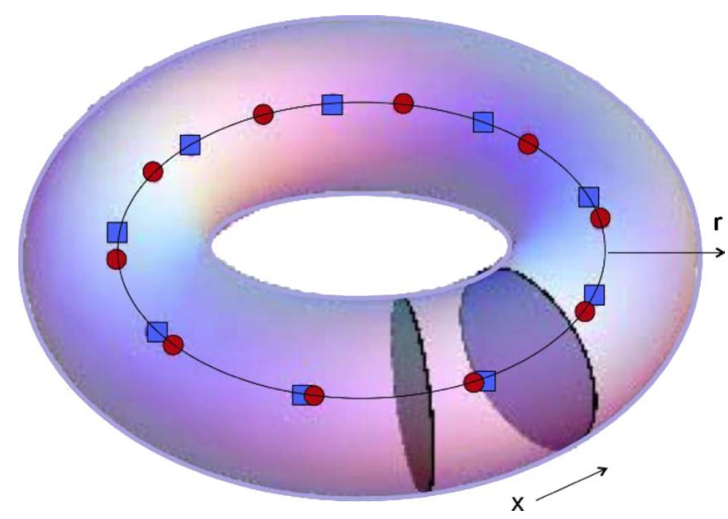

FIG. 13. (Color online) Stern's construction. A large number, $N$, of positive magnetic monopoles (red circles) and $N-1$ negative monopoles (blue squares) uniformizes the radial magnetic field, as shown in Fig. 14. However, the internal flux which is measured through the disks which cut through the torus, oscillates wildly as a function of azimuthal direction $x$, as shown in Fig. 15.

Misha Feigelman, Steve Kivelson, Gil Refael, and Ady Stern for useful discussions. Support of the U.S. Israel Binational Science Foundation and Israel Science Foundation are gratefully acknowledged. A.A. and D.P.A. acknowledge Aspen Center For Physics where many of the ideas were conceived. N.L. acknowledges the financial support of the Israel Clore foundation.

\section{APPENDIX: TRANSLATIONAL SYMMETRY BREAKING ON THE CONTINUOUS TORUS}

In Sec. IV we have shown that the ground state of the HCB Hamiltonian Eq. (4) with $0<N_{\phi}<N$ exhibits TSB relative to the lattice periodicity, in both $x$ and $y$ directions. At first thought this is very surprising: the flux per plaquette is uniform, so one might expect that physical observables in any nondegenerate state would not distinguish one lattice point over another. However, one empirically finds, for example for $N_{\phi}=1$, that the ground state current circulates around a preferred position, $\boldsymbol{R}_{\mathrm{V}}$.

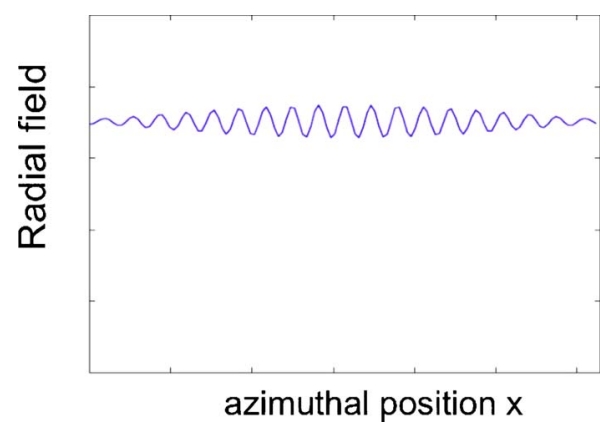

FIG. 14. (Color online) The radial component of the magnetic field penetrating the surface of the torus on the circle $y=0$, as a function of the azimuthal coordinate $x$. The torus contains a ring of $N=20$ monopoles and $N=19$ anti-monopoles, as shown in Fig. 13 . The magnitude of the oscillations decay with increasing $N$, leading to a uniform radial field. 


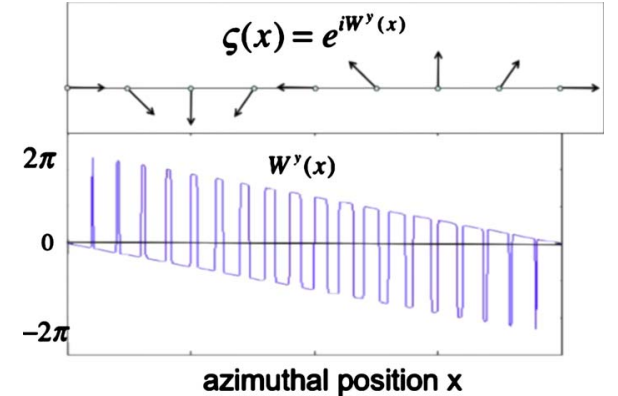

FIG. 15. (Color online) Wilson loop function $W_{y}(x)$, and the phase function $\zeta(x)$, for the monopole configuration of Fig. 13 for $N=20$. While $W_{y}$ oscillates wildly at large $N$ and does not converge to a limit, the physically relevant function $\zeta$ is continuous for all $N$ and breaks translational symmetry.

In this appendix we first explain the reason for the TSB. By working out a specific proposal for trying to construct a purely radial magnetic field, we learn why TSB is in fact unavoidable on the torus.

In the particular choice of gauge field in Sec. IV, the gauge invariant Wilson loop functions $W_{x}, W_{y}$ defined in Eq. (41) are linear functions of $x$ and $y$ modulo $2 \pi$. Therefore, by construction they break translational symmetry in both directions, and we use them to define the special null point on the torus, $\boldsymbol{R}_{0}$.

Now we show that by Stoke's theorem, if $\boldsymbol{A}$ is continuous on any interval $\left[x_{1}, x_{2}\right]$, then $W_{y}(x)$ must be piecewise linear,

$$
W_{y}\left(x_{2}\right)-W_{y}\left(x_{1}\right)=q B L_{y}\left(x_{2}-x_{1}\right),
$$

where $L_{y}$ is the circumference of the torus in the $y$ direction. $\boldsymbol{A}(\boldsymbol{r})$ and $W_{y}(x)$, however, cannot be continuous everywhere on the torus since $W_{y}\left(x_{2}\right)$ must be periodic for $x_{2} \rightarrow x_{2}+L_{x}$ which is inconsistent with a continuous linear behavior given by (A1).

The magnetic field of $N_{\phi}=1$ enters the torus and must end in a magnetic charge. By Dirac quantization, ${ }^{54}$ "magnetic charge" density must be quantized as $\rho_{m}=g_{m} \delta\left(\boldsymbol{r}-\boldsymbol{r}_{m}\right)$ where,

$$
g_{\text {mon }}=1 /(2 q) \text {. }
$$

Therefore the physical reason for the TSB is the pointlike discreteness of the magnetic charge. Consider an embedding of the torus in three dimensions. Dirac quantization forces one to choose a special location $\boldsymbol{r}_{m}$ inside the torus which breaks the translational symmetries.

A possible counter argument is raised: could TSB be avoided by somehow smearing the monopole charge inside the torus? This would presumably restore translational symmetry, at least for the direction in which the embedding has axial symmetry. It is somewhat surprising that the answer is negative, as the particular construction below demonstrates.

Ady Stern $^{55}$ has suggested the following construction. Consider a large number $N$ of positive Dirac monopoles and $N-1$ negative monopoles placed on the middle circle inside the torus, as shown in Fig. 13. One arranges both positive and negative monopoles to be at equal distances between them such that their mean density is uniform. Let us calculate the magnetic fields and their effects on bosons on the torus surface, and then take $N$ to infinity.

For large $N$, the distribution of monopoles approaches a uniform density with total monopole charge of $g_{m}$. We compute numerically the magnetic field created by $N=20$ monopoles situated on rings of radius $R$, as given by Coloumb's law

$$
\mathbf{B}=\frac{\boldsymbol{r}}{2 q r^{3}} .
$$

The radial magnetic field penetrating the surface of the torus on the circle $y=0$ (in the plane of the monopole ring), becomes increasingly closer to a constant, with decreasing oscillatory component as shown in Fig. 14. This behavior is precisely analogous to the electric field from a ring with uniform charge density.

Now let us examine the function $W_{y}(x)$, evaluated for the same monopole configuration as Fig. 15. By Gauss's law, it exhibits discontinuous jumps of size $2 \pi(-2 \pi)$ at each position where the cross section of the torus at $x$ cross through a monopole (antimonopole). Note that $W_{y}(x)$ exhibits $N$ positive jumps and $N-1$ negative jumps, corresponding to the number of positive and negative monopoles. Therefore, in order for $W_{y}(x)$ to be periodic in $x \rightarrow x+L_{x}$, the continuous part of $W_{y}(x)$ needs to compensate for the extra positive jump. Indeed, as can be seen by Fig. 15, between the $2 N$ -1 jumps $W(x)$ decreases linearly, as demanded by Eq. (A1).

This increasingly discontinuous function does not converge to a well-defined limit function in the large $N$ limit. However, the physically relevant function which effects the dynamics of our bosons of charge $q$ on the surface is the unimodular phase function

$$
\zeta(x)=e^{i W_{y}(x)}=\zeta_{0} e^{i q B L_{y} x}, \quad x \in\left[0, L_{x}\right],
$$

which is perfectly continuous and periodic on the circle. Here we see that $\zeta\left(x_{0}\right)=1$ uniquely defines a special position $x_{0}$ which breaks lattice translational symmetry.

We note that the values of $\zeta(x)$ have physical consequences on the current distribution. In the ground state, the loops in the region of $\zeta \approx 1$ feel a weak $\mathrm{AB}$ flux, and thus a relatively weak persistent current is induced in these regions. Similarly, the persistent currents are expected to be maximized around loops with $\zeta \approx-1$.

Thus we learn that a static configuration of monopoles leads translational symmetry breaking. However, if one considers the possibility of an extended quantum wave function of a monopole, the magnetic field will be in a quantum superposition. In this case, translational symmetry can be restored in the entangled state of the matter field with the electromagnetic field. 
${ }^{1}$ L. Pitaevskii and S. Stringari, Bose Einstein Condensation (Oxford University Press, New York, 2003).

${ }^{2}$ D. Jaksch and P. Zoller, Ann. Phys. 315, 52 (2005).

${ }^{3}$ A. van Oudenaarden and J. E. Mooij, Phys. Rev. Lett. 76, 4947 (1996).

${ }^{4}$ E. Altman and A. Auerbach, Phys. Rev. Lett. 81, 4484 (1998).

${ }^{5}$ R. Fazio and H. van der Zant, Phys. Rep. 355, 235 (2001).

${ }^{6}$ M. P. A. Fisher, P. B. Weichman, G. Grinstein, and D. S. Fisher, Phys. Rev. B 40, 546 (1989).

${ }^{7}$ Y. J. Uemura, G. M. Luke, B. J. Sternlieb, J. H. Brewer, J. F. Carolan, W. N. Hardy, R. Kadono, J. R. Kempton, R. F. Kiefl, S. R. Kreitzman, P. Mulhern, T. M. Riseman, D. Ll. Williams, B. X. Yang, S. Uchida, H. Takagi, J. Gopalakrishnan, A. W. Sleight, M. A. Subramanian, C. L. Chien, M. Z. Cieplak, G. Xiao, V. Y. Lee, B. W. Statt, C. E. Stronach, W. J. Kossler, and X. H. Yu, Phys. Rev. Lett. 62, 2317 (1989).

${ }^{8}$ V. J. Emery and S. A. Kivelson, Nature (London) 374, 434 (1995).

${ }^{9}$ T. Kostyrko and J. Ranninger, Phys. Rev. B 54, 13105 (1996).

${ }^{10}$ A. Paramekanti, M. Randeria, T. V. Ramakrishnan, and S. S. Mandal, Phys. Rev. B 62, 6786 (2000); H. J. Kwon, A. T. Dorsey, and P. J. Hirschfeld, Phys. Rev. Lett. 86, 3875 (2001).

${ }^{11}$ E. Altman and A. Auerbach, Phys. Rev. B 65, 104508 (2002).

${ }^{12}$ M. Franz and A. P. Iyengar, Phys. Rev. Lett. 96, 047007 (2006); I. F. Herbut and M. J. Case, Phys. Rev. B 70, 094516 (2004).

${ }^{13}$ A. Mihlin and A. Auerbach, Phys. Rev. B 80, 134521 (2009).

${ }^{14}$ A. L. Fetter and J. D. Walecka, Quantum Theory of ManyParticle Systems (McGraw-Hill, New York, 1971), Chap. 6.

${ }^{15}$ N. H. Lindner and A. Auerbach, Phys. Rev. B 81, 054512 (2010).

${ }^{16}$ V. J. Emery and S. A. Kivelson, Phys. Rev. Lett. 74, 3253 (1995).

${ }^{17}$ N. H. Lindner, A. Auerbach, and D. P. Arovas, Phys. Rev. Lett. 102, 070403 (2009).

${ }^{18}$ We note that in the case of half filled fermion bands, particlehole conjugation is obtained by sending $f_{i} \rightarrow f_{i}^{\dagger}$. However, due to the anticommutation of fermion operators, Hamiltonians can realize this symmetry only on bipartite lattices. In contrast, particle-hole symmetry of half-filled HCB models occurs on any graph.

${ }^{19}$ A. Auerbach, Interacting Electrons and Quantum Magnetism (Springer-Verlag, Berlin, 1994), Chap. 13.

${ }^{20}$ A. Auerbach, arXiv:cond-mat/9801294 (unpublished).

${ }^{21}$ A. W. Sandvik and C. J. Hamer, Phys. Rev. B 60, 6588 (1999).

${ }^{22}$ K. Bernardet, G. G. Batrouni, J.-L. Meunier, G. Schmid, M. Troyer, and A. Dorneich, Phys. Rev. B 65, 104519 (2002).

${ }^{23}$ V. L. Berezinskii, Sov. Phys. JETP 34, 610 (1972); J. M. Kosterlitz and D. J. Thouless, J. Phys. C 5, L124 (1972).

${ }^{24}$ A. Auerbach, D. P. Arovas, and S. Ghosh, Phys. Rev. B 74, 064511 (2006).
${ }^{25}$ D. P. Arovas and A. Auerbach, Phys. Rev. B 78, 094508 (2008).

${ }^{26}$ F. D. M. Haldane, Phys. Rev. Lett. 61, 1029 (1988).

${ }^{27}$ C. Wu, H. D. Chen, J. P. Hu, and S.-C. Zhang, Phys. Rev. A 69, 043609 (2004); H.-D. Chen, O. Vafek, A. Yazdani, and S.-C. Zhang, Phys. Rev. Lett. 93, 187002 (2004).

${ }^{28}$ F. D. M. Haldane and Y. Wu, Phys. Rev. Lett. 55, 2887 (1985).

${ }^{29}$ F. D. M. Haldane and E. H. Rezayi, Phys. Rev. B 31, 2529 (1985).

${ }^{30}$ D. P. Arovas, R. N. Bhatt, F. D. M. Haldane, P. B. Littlewood, and R. Rammal, Phys. Rev. Lett. 60, 619 (1988).

${ }^{31}$ R. Tao and F. D. M. Haldane, Phys. Rev. B 33, 3844 (1986).

${ }^{32}$ J. E. Hoffman, E. W. Hudson, K. M. Lang, V. Madhavan, H. Eisaki, S. Uchida, and J. C. Davis, Science 295, 466 (2002).

${ }^{33}$ L. Balents, L. Bartosch, A. Burkov, S. Sachdev, and K. Sengupta, Phys. Rev. B 71, 144508 (2005); 71, 144509 (2005).

${ }^{34}$ E. T. Whittaker and G. N. Watson, A Course of Modern Analysis (Cambridge University Press, Cambridge, 1999).

${ }^{35}$ D. P. Arovas (unpublished).

${ }^{36}$ M. P. A. Fisher and D.-H. Lee, Phys. Rev. B 39, 2756 (1989).

${ }^{37}$ D. P. Arovas and J. A. Freire, Phys. Rev. B 55, 1068 (1997).

${ }^{38}$ C.-C. Joseph Wang, R. A. Duine, and A. H. MacDonald, Phys. Rev. A 81, 013609 (2010).

${ }^{39}$ S. Doniach and B. A. Huberman, Phys. Rev. Lett. 42, 1169 (1979).

${ }^{40}$ D. S. Fisher, Phys. Rev. B 22, 1190 (1980).

${ }^{41}$ W. R. Magro and D. M. Ceperley, Phys. Rev. Lett. 73, 826 (1994).

${ }^{42}$ J. E. Avron and R. Seiler, Phys. Rev. Lett. 54, 259 (1985).

${ }^{43}$ G. D. Mahan, Many-Particle Physics (Plenum, NY, 1981).

${ }^{44}$ We note that a finite superfluid stiffness has been obtained for the CB phase by H. Nordborg and G. Blatter, Phys. Rev. Lett. 79, 1925 (1997).

${ }^{45}$ M. V. Feigelman, V. B. Geshkenbein, L. B. Ioffe, and A. I. Larkin, Phys. Rev. B 48, 16641 (1993).

${ }^{46}$ A. S. Sørensen, E. Demler, and M. D. Lukin, Phys. Rev. Lett. 94, 086803 (2005).

${ }^{47}$ M. Hafezi, A. S. Sørensen, M. D. Lukin, and E. Demler, EPL 81, 10005 (2008).

${ }^{48}$ C. Lannert, M. P. A. Fisher, and T. Senthil, Phys. Rev. B 63, 134510 (2001).

${ }^{49}$ Z. Tešanović, Phys. Rev. Lett. 93, 217004 (2004).

${ }^{50}$ A. Melikyan and Z. Tesanovic, Phys. Rev. B 71, 214511 (2005).

${ }^{51}$ H. Takagi, T. Ido, S. Ishibashi, M. Uota, S. Uchida, and Y. Tokura, Phys. Rev. B 40, 2254 (1989).

${ }^{52}$ H. Y. Hwang, B. Batlogg, H. Takagi, H. L. Kao, J. Kwo, R. J. Cava, J. J. Krajewski, and W. F. Peck, Jr., Phys. Rev. Lett. 72, 2636 (1994).

${ }^{53}$ F. Gerbier and J. Dalibard, arXiv:0910.4606 (unpublished).

${ }^{54}$ P. A. M. Dirac, Proc. R. Soc. London, Ser. A 133, 60 (1931).

${ }^{55}$ A. Stern (private communication). 